\section{Trends in Physics}

\section{EPS 6th General Conference, 27-31 August 1984}

Why are the General Conferences of the European Physical Society so special? There are a number of reasons:

1) As physics becomes more and more specialised, the opportunities for obtaining a bird's eye view of developments in physics outside one's own particular discipline become more and more restricted.

2) Parallel to the specialisation there is a greater inter-dependence of disciplines as the techniques, both theoretical and experimental, that have been developed in one discipline become relevant to another.

3) Even the data generated in one field may have an immediate impact on another but there is always the risk that they remain unperceived.

4) Few areas of physics can retain a peak interest indefinitely: some will fall as others rise and it is neither productive nor intellectually rewarding to pursue in ever greater detail - or persuade students to follow the minutiae of a subject that has already been worked through.

5) Although many good review journals are published, the quantity of material that has to be read immediately leads to neglect of long-term information.

6) Because of mounting pressures, not least those of administration, the time for reflective and relaxed exchanges with colleagues from far afield becomes shorter. 7) International exchange, recognized everywhere as an invaluable stimulus, is so often confined to exchanges within a small circulating group that has less and less input from outside.

8) The generation gap is not merely a domestic problem but intellectual also, perpetuated often by the traditional structures that have grown up.

The EPS General Conferences are designed to counter these tendencies. Almost unique in the physics meetings calendar, they provide a genuine over-view of the high-points in physics through the Plenary Sessions and a more detailed insight into subjects of outstanding topicality through the Symposia. Nor is the impact of physics on the well-being of mankind ignored, with special sessions devoted to educational and social problems. A generous participation of young physicists is encouraged through the support given by the EPS Young Physicists Fund and the different schemes operated by the various national societies.

Prague is a magnificient location and the Agricultural Faculty where the Conference will be held is particularly attractive, with wide lawns and flowers beds, ponds and fountains - a campus that combines modern buildings and facilities with a parklike charm. Altogether a setting which should provide the ideal conditions for maximum interchange. Because, as we are all aware, it is not just the Conference sessions, it is the interaction of minds in an intellectually stimulating yet relaxed environment which can bring so much to the participants and have such an important long-term impact on their work.

The first bulletin produced by the International Programme Committee under its chairman, Professor Leon Van Hove has now been published and is available together with preliminary registration forms at the EPS Secretariat. Members of EPS receive their individual copies with this issue of Europhysics News.

\section{Guide to Entries}

Europhysics News publishes annually three Meetings Issues: in March, June and November. In the March issue the information received to date is given in full; in the two subsequent issues some entries may be abbreviated and marked by an asterisk $\left({ }^{*}\right)$ indicating that full information can be found in the preceding Meetings Issue of Europhysics News.

The events listed are those for which details were received at the EPS Secretariat in Geneva by May 1983. In general, all events interesting European physicists which take place from six weeks after the date of publication are quoted. Most readers will already have taken their decision regarding earlier events but like to have as long an advance notice as possible of future happenings.

The listings are in date order divided into two categories - Conferences and Schools. Europhysics Conferences, i.e. meetings organized by EPS, its Divisions and Sections, are printed in bold type and boxed; events sponsored by EPS are printed in bold type; Europhysics Study Conferences are marked by an $\Theta$ preceding the date.

For inclusion in the November 1983 issue, organizers should send available details to the EPS Secretariat by 17 October.

Organizers of conferences that are European in concept may apply for EPS sponsorship through the Secretariat.

\section{Key to Abbreviations}

A: Application deadline

Ab: Abstracts deadline

inv.: restricted to invited applicants

NP: no proceedings will be published

$P$ : Papers deadline (information on proceedings has not been received)

PP: proceedings will be published.

The information is given in this order:

Date

Venue

Title

Contact for information

$\mathrm{A}:$.... / Ab: .... / PP, NP or P: Papers deadline / Number of participants / inv. / languages other than English / Sponsorship / Conference fee.

4 after the date indicates that there has been an important amendment to a previously published entry. 
For Guide to Entries see Front Page

\section{Conferences 1983}

\section{0-27 July \\ Int. Europhysics Conf. on Highton, UK Physics \\ J.J. Thresher, Rutherford Appleton Lab., Chilton, Didcot, Oxon OX11 00X \\ A: 1.3.83 / Ab: 20.5.83 / PP: 20.6.83 / $800 /$ inv. / f 80. - IOM; 90. - mbrs 4b; 40. - students}

\section{4-25 July}

nr. Rome, Italy

* Wave Functions and Mechanism from Electron Scattering Processes

24-25 July

- Collisions in Strong Fields

Bielefeld, FRG

24-26 July

Münster, FRG

- Polarization \& Correlation in Electron-Atom Collisions

24-29 July

Albany, NY, USA

- 9th AIRAPT International High-Pressure Conf.

25-26 July

Berlin-West

- Int. Workshop on Atomic and Molecular Photoionization (Use of Synchrotron Radiation)

25-29 July

Trieste, Italy

- Computer Simulation of Quantum Systems

25-29 July

Edinburgh, UK

- Statistical Mechanics (STAT - PHYS 15)

27 July - 2 Aug.

Berlin-West

* 13th Int. Conf. on Physics of Electronic and Atomic Collisions (ICPEAC)

31 July - 4 Aug.

New York, NY, USA

- American Assoc. of Physicists in Medicine

1-4 Aug. Aspen, CO, USA

* 2nd Topical Meeting on Coherent Laser Radar

1-5 Aug.

St. Andrews, UK

- 75th Jubilee Conference on Helium-4

1-10 Aug.

Boulder, CO, USA

* 7th Int. Conf. on Mathematical Physics

4-5 Aug. Kaiserslautern, FRG

- 3rd Symp. on Dynamics of Molecular Collisions

4-5 Aug.

nr. Utrecht, NL

* Int. Seminar on lon-Atom Collisions (ISIAC)

4-5 Aug.

Innsbruck, Austria

* International Swarm Seminar

Linz, Austria

- 3rd Workshop: Inner - Shell lonization by Light lons

4-9 Aug. Ithaca, NY, USA

- Lepton Photon Interaction at High Energies

College Park, MD, USA
- Unstable Current Systems and Plasma Instabilities in

College Park, MD, USA Astrophysics (IAU)

8-12 Aug.

Jerusalem, Israel

7th Int. Conf. on Vacuum Ultraviolet Radiation Physics

I.T. Steinberger, Racah Inst. of Physics, Hebrew University, Jerusalem

A: Spring 83 / PP: Spring 83 / 400 / IUPAP / approx. US $\$ 130 .-$ incl. proc.

8-12 Aug. Liège, Belgium

-8th European Crystallographic Meeting

11-16 Aug.

-12th Int. Conf. on High Energy Accelerators
15-19 Aug.

Colorado Springs, CO, USA

Cryogenic Engineering Conf. - Int. Cryogenic Materials Conf

T.M. Flynn, 773.1, National Bureau of Standards, 1-4001, 325 Broadway, Boulder, CO 80303

A: $15.8 .83 / \mathrm{Ab}: 1.3 .83 / \mathrm{PP}: 15.8 .83 / 600$

\section{7-19 Aug.}

Lund, Sweden

Atomic Spectra and Oscillator Strengths for Astrophysics and Fusion Research

Atomic Spectroscopy, Physics Dept., Univ. of Lund, Sölvegatan 14, S-22362 Lund

18-26 Aug. Lyngby/Copenhagen, DK

- IUPAC General Assembly

21-27 Aug.

Karlsruhe, FRG

10th Int. Conf. on Few-Body Problems in Physics

22-26 Aug.

Stuttgart, FRG

* 4th Int. Conf. on Phonon Scattering in Condensed Matter

22-26 Aug.

Tokyo, Japan

10th Int. Conf.: Amorphous and Liquid Semicond.

22 Aug. - 3 Sept.

- 18th Int. Cosmic Ray Conference

Bangalore, India

24-26 Aug.

- Thermal Analysis and Calorimetry

29-30 Aug.

Workshop on Search for Heavy Flavours

Como, Italy

29-31 Aug.

- MECO 83: Measurement and Control

6th IASTED Int. Symposium

29 Aug. - 2 Sept.

- Computer Coupling of Phase Diagrams and Thermochemistry (CALPHAD XII)

29 Aug. - 2 Sept.

Delft, NL

Atmospheric Dispersion of Heavy Gases and Small Particles

G. Ooms, Lab. for Aero- and Hydrodynamics, Dept. of Mechanical Engineering, Delft Univ. of Technology, Rotterdamseweg 145, NL-2628 AL Delft

inv. / IUTAM, IUGG

29 Aug. - 3 Sept.

- Int. Conf. on Nuclear Physics

Florence, Italy

29 Aug. - 3 Sept. Düsseldorf, FRG
16th Int. Conf. on Phenomena in lonized Gases

29 Aug. - 3 Sept. Düsseldorf, FRG
16th Int. Conf. on Phenomena in Ionized Gases th Int. Conf. on
(ICPIG - XVI)

K. Suchy, Inst. f. Theor. Physik II, Universität Düsseldorf,

Universitätsstr. 1, D-4000 Düsseldorf 1

PP: 8.4 .83 / 500 / IUPAP / approx. DM 220. - incl. proc.

29 Aug. - 3 Sept.

Altenberg, FRG

* 3rd Int. Conf. on Recent Progress in Many-Body Theories

30 Aug. - 2 Sept. Asiago, Italy

* Astronomy with Schmidt-Type Telescopes (IAU)

30 Aug. - 2 Sept.

Guildford, UK

* EMAG' 83

31 Aug. - 2 Sept.

Lake Como, Italy

Physics in Collisions III: High Energy ee, ep, pp Interactions

G. Bellini, Ist. di Scienze Fisiche "Aldo Pontremoli", Via Celoria 16, 1-20133 Milano

A: 15.5 .83 / PP / 150 / inv. / US \$ 85. - incl. proc.

1-4 Sept.

Bucharest, Romania

Magnetism of Rare Earths and Actinides

E. Burzo, Central Inst. of Physics,

POB MG-6, Bucharest-Magurele

A: 1.8.83 / PP: 15.6.83 / 250 / incl. proc. SFr. 100. IOM; 200. - others

4-6 Sept.

Herrsching, Ammersee, FRG

Techniques in Studies of Biological Effects of Low Level Millimetre Waves

F. Keilmann, Max-Planck Inst. f. Festkörperforschung, D-7000 Stuttgart 80

Ab: 30.6 .83 / NP / 40 / URSI

5-8 Sept.

Tübingen, FRG

Structure

anic Clouds

5-8 Sept.

Orsay, France

- Int. Symp.: Highly Excited States - Nuclear Structure
5-9 Sept.

London, UK

Advanced Technology Optical Telescopes II (5-6.9) Instrumentation in Astronomy V (7-9.9)

SPIE, POB 10, Bellingham, WA 98227-0010, USA

5-9 Sept.

- Magnet Technology 8 (MT-8)

Grenoble, France

5-9 Sept.

Durham, UK

* Int. Conf. on Fourier Transform Spectroscopy

5-9 Sept.

Toulouse, France

- 10th Int. Conf.: Optics of X-Rays - Microanalysis

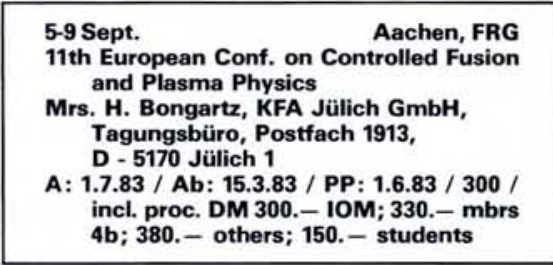

5-9 Sept.

Bucharest, Romania

-29th Int. Symposium on Macromolecules

5-10 Sept.

Trieste, Italy

* 12th Int. Coll, on Group Theor. Methods in Physics

6-9 Sept.

- 2nd Int. Conference on Lasers

Peking, P.R. of China

6-9 Sept.

rials 6 (SMM6)

K. Tompa, Central Res. Inst. f. Physics, POB 49, H-1525 Budapest

A: 15.12.82 (pre-reg.) / PP / 200 / inv. /

US $\$ 95 .-$ IOM, mbrs 4b; 105.- others;

25. - students

\section{6-13 Sept.}

Bratislava, CSSR

11 th Int. Symposium on Nuclear Electronics

P. Povinec, Comenius Univ., Dept. of Nuclear Phys. Mlynska dolina F-1, CS-84125 Bratislava

Ab: 15.3.83 / PP / 150 / Engl., Russian / Kcs 600. incl. proc.

7-9 Sept.

Ferrara, Italy

A. Conf, on Magnetic Recording Media

A. Cecchetti, Ist. di Fisica, Università di Ferrara,

Via Paradiso 12, 1-44100 Ferrara

A: 1.3.83 / Ab: 1.4 .83 / PP: 28.5 .83 / 40 / US \$170.-

7-17 Sept.

Varenna, Italy

Course and Workshop: Mirror-Based and

Field-Reversed Approaches to Magnetic Fusion

11-16 Sept.

Antwerpen, Belgium

- Joint Meeting: Deutsche Ges, f. Elektronenmikroskopie, Soc. Belge de Microscopie Electronique

12-14 Sept.

BNL, Upton, NY, USA

- 3rd Nat. Conf. on Synchrotron Rad. Instrumentation

12-14 Sept.

San Francisco, CA, USA

- 5 th Int. Conf. on High Power Particle Beams

12-15 Sept.

Villigen, Switzerland

- Magnet Measurement Workshop (MMW-3)

12-16 Sept.

Kyoto, Japan

- Int. Ion Engineering Congress (ISIAT 83, IPAT 83)

12-16 Sept.

Geneva, Switzerland

- Observational Tests of the Stellar Evolution Theory

12-16 Sept.

- Int. Conf. on Dynamics of Interfaces

Lille, France

\section{2-16 Sept.}

7th Int. Conf. on Crystal Growth

Stuttgart, FRG

K.W. Benz, Secretary ICCG-7, Kristall-Labor, Physikalisches Institut der Universität, Pfaffenwaldring 57, D-7000 Stuttgart - 80

A: 1.8 .83 / Ab: 1.3 .83 / PP: 1.3 .83 / 650 / IUPAC, IUPAP, IUCr / DM 240, - before 1.8.83

12-16 Sept.

Lauzelle (Ottignies), B

* 16th Int. Symposium on Free Radicals 


\section{2-17 Sept.}

Heraklion, Crete 6th Specialized Int. Colloque Ampère: Quadruple Interactions and Spatially Resolved NMR in Solids

\section{3-15 Sept. 4}

Surface Analysis ' 83

Conf. Secr., Dom Techniky,

Skultétyha 1, CS-83227 Bratislava

Ab: 1.3.83 / P: 1.3.83 / Engl., Russian

\section{3-15 Sept.}

Trieste, Italy

Clusters and Groups of Galaxies

F. Mardirossian, Osservatorio Astronomico di Trieste, Via G.B. Tiepolo 11, 1-34131 Trieste

A: 15.8.83 / Ab: 30.6.83 / PP: 15.8.83/100/ US $\$ 50$. -

\section{3-16 Sept. Canterbury, UK \\ 13th European Solid State Device Research Conf. (ESSDERC) \\ 8th Symposium on Solid State Device Technology (SSSDT) \\ Meetings Officer, Institute of Physics, 47 Belgrave Square, London SWIX 8 OX \\ Ab: 2.5.83 / PP / 300 / f 95. - IOM, mbrs $4 b ; 115 .-$ others, incl. proc.; 47 . - stu- dents}

13-16 Sept. Strasbourg, France

Statistical Methods in Astronomy

C. Jaschek, Observatoire de Strasbourg,

11 rue de l'Université, F-6700 Strasbourg

14-16 Sept.

Orléans, France

Collisions et Rayonnement

P. Ranson, GREMI, Univ. d'Orléans,

F-45046 Orléans Cedex

14-16 Sept.

Loughborough, UK

- Concentrated Colloidal Dispersions

14-16 Sept.

Madrid, Spain

9th Symposium on Microprocessing and Microprogramming (Euromicro 83): Developments in Industry, Business and Education

Euromicro, TH Twente, POB 217, NL-7500 AE Enschede
14-16 Sept.

- Physical Aspects of Polymer Science

Reading, UK

15-17 Sept.

Blacksburg, VA, USA

Meeting of the Particles and Fields Div. (APS)

A. Abashian, Physics Dept., Virginia Polytechnic Inst. and State University, Blacksburg, VA 24061

16-18 Sept.

Peking, P.R. of China

-7th Rare Earth - Cobalt Magnet Workshop

18-22 Sept.

Lisbon, Portugal

1st Iberian Symposium on Condensed Matter

18-22 Sept.

Hvar, Yugoslavia

- Rapid Variability of Early-Type Stars

18-23 Sept.

New Delhi, India

12th World Energy Conference

19-21 Sept.
Hungarian Conf. on Crystal Growth

19-22 Sept.

udapest, Hungary

Upton, NY, USA

OSA/BNL Topical Meeting: Free Electron Generation of Extreme Ultraviolet Coherent Radiation

Optical Society of America, 1816 Jefferson Place, N.W., Washington, DC 20036

$A b: 20.6 .83 / P: 12.9 .83 /$ before 19.8: US $\$ 140 .-$ $50 .-$ students; at meeting $160,-, 65 .-$

19-22 Sept.

6th Nat. Quantum Electronics Conf.

19-23 Sept.

Sussex Univ., UK

Physical Chemistry of the Solid State - Applications to Metals and Their Compounds

19-23 Sept. Conf. on Engineering Rheology - Meeting of European Rheologists

19-23 Sept.

8th Coll. High Resolution Molecular Spectroscopy

19-24 Sept.

Grenoble, France

- Congrès Général de la Société Française de Physique
20-22 Sept.

Manchester, UK

Sensors and their Applications

Budapest, Hungary

3rd Conf of Technical Committee on ment (FLOMEKO '83)

20-23 Sept.

Lausanne, Switzerland th European Solid-State Circuits Conf. (ESSCIRC ' 83 ) V. Valencic, ESSCIRC 83, EPFL, 33 ave. de Cour, $\mathrm{CH}-1007$ Lausanne

A: 1.8.83 / Ab: 25.4.83 / PP / SFr. 200.- ; $80,-$ students

\section{0-23 Sept.} Berlin-West

Computing in Accelerator Design (was Warsaw 1982)

W. Busse, Hahn-Meitner-Inst. f. Kernforschung, Glienicker Str. 100, Postfach 390128, D-1000 Berlin 39 A : 1.6.83 / Ab: 1.5.83 / PP: 20.9.83 / DM $300 .-; 50 .-$ students

20-23 Sept. Hamburg, FRG 15th Europhysics Conf. on Macromolecu lar Physics and

Hamburg Macromolecular Symp. on New Developments in the Characterization of Polymers in the Solid State

H.G. Zachmann, Abt. Angewandte Chemie, Univ. Hamburg, Martin-LutherKing-Platz 6, D-2000 Hamburg

A: $1.8 .83 / \mathrm{Ab}: 30.4 .83 / \mathrm{NP} / 150$ / DM 200. - IOM, mbrs. $4 b$, 240. - others, $40 .-$ students

20-26 Sept.

Sant Feliu de Guixols, E

1 st Int. Meeting on the History of Scientific Ideas: Sym metries in Physics 1600-1980

M.G. Doncel, Dept. Fisica Teorica, U.A.B.

Bellaterra (Barcelona)

A: 30.6 .83 / Ab: 30.6 .83 / PP: $20.9 .83 / 100$ US $\$ 100$. -

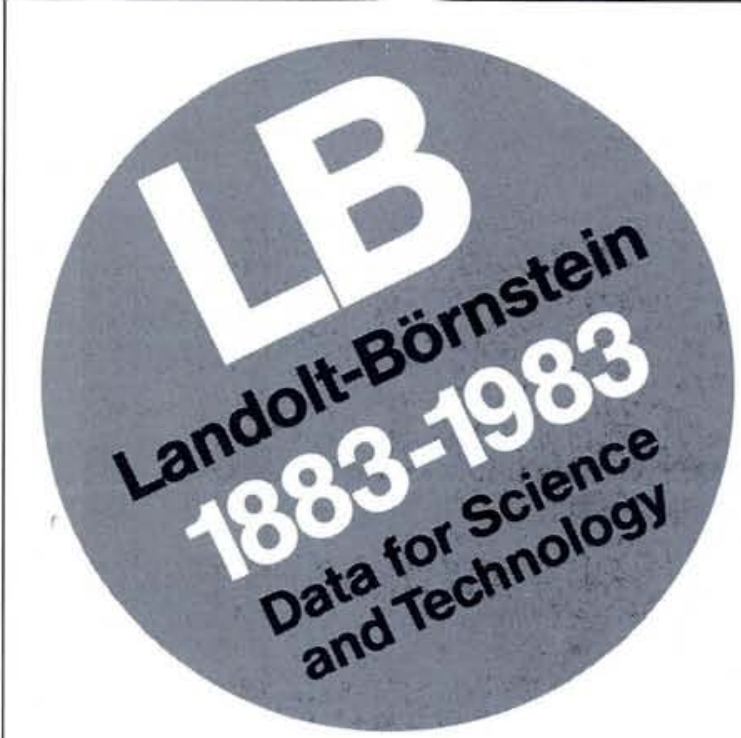

Please send for the following

information material (free of charge):

Landolt-Börnstein Outline

(list of all available volumes),

Guide to Landolt-Börnstein Data

(a reference chart, $70 \times 100 \mathrm{~cm}$ ),

What is Landolt-Börnstein?

(an informative brochure).
Landolt-Börnstein provides a systematic, up-to-date, and comprehensive collection of reliable numerical data and functional relationships in text, tabular, and graphical form from all fields of Physics, Physical Chemistry, Geophysics, Astrophysics, and Technology.

The data are critically evaluated and selected from the original literature (cited in each case) by leading scientists from all over the world.

Landolt-Börnstein celebrates in 1983 a century of service to science and technology.

\section{Springer-Verlag Berlin Heidelberg New York}

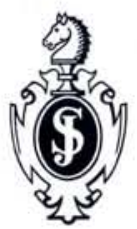

4005-Marketing, Heidelberger Platz 3, D-1000 Berlin 33 Editorial Office, Schuchardstrasse 10, D-6100 Darmstadt 
26-28 Sept. 4

Hertzian Optics and Dielectrics

Y. Servant, Lab. d'Optique Ultra-Hertzienne, Université de Bordeaux-I, F-33405 Talence Cedex

A : 30.6.83 / Ab: 28.5.83 / PP: 26.9.83 / 100 / Engl. French / FF 250. - incl. proc.

26-29 Sept.

Oxford, UK

Anniversary Meeting: Office Automation

C.J.M. Aarts, SEAS Headquarters,

Toernooiveld, NL-6525 ED Nijmegen

A: 1.9.83 / Ab: 15.5.83 / PP: 1.9.83/300/ hFI. 1000.-

26-29 Sept.

Upton, NY, USA

3rd Int. Conf. on Ultrarelativistic Nucleus-Nucleus Collisions

Mrs. R. Greenberg, Physics Dept., Bldg 510A Brookhaven Nat. Lab., Upton, NY 11973

26-30 Sept. London, UK

-16th European Conf, on Laser Interaction with Matter

26-30 Sept.

Liblice, CSSR

- Int. Symposium on Jahn-Teller Effect

26 Sept. - 1 Oct. Torremolinos (Malaga), Spain 5th European Meeting on Ferroelectricity EMF 5

E. Maurer, Instituto de Fisica de Materiales, Serrano 144, E-Madrid 6

A: 1.7.83 / Ab: 1.3.83 / PP: 26.9.83 / 500 /

US $\$ 100 .-; 75 .-$ students

26 Sept. - 1 Oct.

Madrid, Spain

Congress (IX IVC

5 th Int. Conf. on Solid Surfaces (V ICSS)

IX IVC - V ICSS Secr., Inst. de Fisica de Materiales, CSIC, Serrano 144, Madrid-6

A: 20.7 .83 / Ab: 1.4 .83 / IUVSTA / US $\$ 240$.-

26 Sept. - 7 Oct.

Murcia, Spain

$4^{\circ}$ Reunion del Grupo de Electricidad y Magnetismo

P.E.M. Rodriguez, Catedra de Electricidad

Facultad de Ciencias, Murcia

27-29 Sept.

Hamburg, FRG

DESY Workshop on Hadron and Jet Structure

K. Schilling, Fachber. Physik, Gesamthochschule Wuppertal, Gauss-Str. 20, D-5600 Wuppertal

\section{7-30 Sept.}

Paris, France

Int. Meeting: Phys, Chemistry of Colloids and Interfaces - Biotechnologies and Drug Research

C. Troyanowsky, Soc. de Chimie Physique, 10 rue Vauquelin, F-75231 Paris Cedex 03

A: $20.9 .83 / \mathrm{Ab}: 1.3 .83 / \mathrm{P}$ : at $\mathrm{mtg}$. / 250

\section{8-30 Sept.}

Nantes, France

Congrès International sur le Comportement mécanique et physique des Matériaux sous sollicitations dynamiques

Chiem-Chi-Yuen, ENSM, 1 rue de la Noë,

F-44072 Nantes Cedex

Ab: $1.3 .83 / \mathrm{PP}$

28-30 Sept.

Jahrestagung der Oesterreichischen Physikalischen Gesellschaft

H. Paul, Inst. f. Experimentalphysik,

Universität Linz, A-4040 Linz

29 Sept. - 1 Oct.

Tokyo, Japan

High Energy Photonuclear Reactions and Related Topics

S. Homma, Inst. f. Nuclear Study, Univ. of Tokyo, Tanashi, Tokyo 188

A: 30.7 .83

1-7 Oct.

Erice, Italy

Workshop on Induced Gravity

S.L. Adler, School of Natural Sciences, Institute of Advanced Study, Princeton, NJ 08540

2-5 Oct.

Oak Brook, IL, USA

Int. Conf. on Thermal Infrared Sensing for Diagnostics and Control (Thermosense VI)

SPIE, POB 10, Bellingham, WA 98227-0010

Ab: $2.5 .83 / P: 1.8 .83$

3-5 Oct.

Capri, Italy

Int. Seminar on Indoor Exposure to Natural Radiation and Related Risk Assessment

Secr. Int. Sem., CEC, J. Sinnaeve DG XII-F-1, rue de la Loi 100, B-1049 Brussels

Ab: $15.3 .83 / P P: 3.10 .83$

4-6 Oct.

3rd Symposium of Tech. Comm. on Technical Diag. nostics

IMEKO Secretariat, POB 457, H-1371 Budapest 5
46 Oct.

Cincinnati, $\mathrm{OH}$, USA

15th National Technical Conf. of SAMPE

M. Smith, SAMPE, POB 613, Azusa, CA, 91702

Ab: $1.2 .83 / P: 1.7 .83$

$470 \mathrm{ct}$.

Toulouse, France

Environmental and Thermal Control Systems for Space Vehicles

CNES, Dépt des Affaires Universitaires,

18 ave, Edouard-Belin, F-31055 Toulouse Cedex Engl., French

5-7 Oct. Toronto, Canada

41st Int. Pittsburgh Diffraction Conf.

3rd Canadian Crystallography Conf.

R.H. Marchessault, Xerox Res. Centre of Canada,

2480 Dunwin Drive, Mississauga, Ont. L5L 1J9

6-9 Oct.

Ann Arbor, MI, USA

Monopole ' 83 Conference

Stone, Monopole '83, Univ. of Michigan, Dept. of Physics, Ann Arbor, Ml 48109

10-13 Oct.

Louvain-la-Neuve, B

Int. Symposium Georges Lemaître (IAU)

A. Berger, Inst. of Astronomy and Astrophysics G. Lemaître, Catholic Univ. of Louvain, chemin du Cyclotron 2, B-1348 Louvain-la-Neuve

10-13 Oct.

Albuquerque, NM, USA

3rd Topical Meeting on Fusion Reactor Materials

M.J. Davis, Dept, 5830 , Sandia Nat, Laboratories, PP POB 5800 , Albuquerque, NM 8718

10-14 Oct.

Trieste, Italy

Perspectives in Nuclear Physics at Intermediate Energies Int. Centre of. Theor. Phys., POB 586, 1-34100 Trieste A: 30.6 .83

10-15 Oct.

Budapest, Hungary

34th Congress of Int. Astronautical Federation

Hungarian Astronautical Society.

Kossuth Lajos tér. 6-8, $\mathrm{H}-1055$ Budapest

11-13 Oct.

Lausanne, Switzerland

Journées d'Electronique de I'EPFL 1983: Testing Complex Integrated Circuits - A Challenge

Secr. Journées d'Electronique EPFL, ch. de Bellerive 16, $\mathrm{CH}-1007$ Lausanne

A: 15.9.83 / Ab: 15.3.83 / PP: 1.8.83 / Engl., French / incl. proc. SFr. $180 .-$ before $15.9 .83 ; 240 .-$ after

11-14 Oct. Albany, NY, USA

36th Annual Gaseous Electronics Conf.

G.A. Farrall, G.E. Res. and Development Center, POB 8, Schenectady, NY 12301

$A b: 29.7 .83$

13-15 Oct.

Notre Dame, IN, USA

Meeting of Nuclear Physics Division (APS)

The American Physical Society. 335 E. 45th., New York, NY 10017

13-16 Oct.

Delémont/Porrentruy, $\mathrm{CH}$

Réunion d'automne de la Société Suisse de Physique

B. Vaucher, Société Suisse de Physique, Inst. de Phy. sique de I'Université, Pérolles, $\mathrm{CH}-1700$ Fribourg Ab: 15.8.83 / PP / 150/Engl., French, German

16-20 Oct.

Bad Stuer, GDR

Int. Workshop on Electroluminescence

G.O. Müller, Akad. d. Wissenschaften d. DDR, Zentralinstitut $f$. Elektronenphysik, Mohrenstrasse 40/41, DDR-1086 Berlin

A: 1.8 .83 / Ab: 15.6 .83 / P: 15.6 .83 / 50 / US $\$ 300$. incl. board, accomm.

17-18 Oct.

Florence, Italy

2nd European Conf. on Integrated Optics (ECIO 2)

G.C. Righini, EROE, Via Panciatichi 64, 1-50127 Firenze

\section{7-19 Oct.}

Upton, NY, USA

Brookhaven Conf.: Advances in Soft - X-Ray Science and Technology

SPIE, POB 10, Bellingham, WA $98227-0010$

$\mathrm{Ab}: 16.5 .83 / \mathrm{P}: 15.8 .83$

17-20 Oct.

New Orleans, LA, USA

Annual Meeting of Optical Society of America

Optical Society of America, 1816 Jefferson Place, N.W., Washington, D-20036

$A b: 10.6 .83 / P P / U S \$ 105$

20-22 Oct.

Blacksburg, VA, USA

Meeting of Particles and Fields Div. (APS

The American Physical Society,

335 E. 45th Str., New York, NY 10017

20-25 Oct.

Madrid and Lisbon

Primeras Jornadas Hispano-Portuguesas de Tribologia A. Rincon, Instituto Rocasolano, Serrano 119, Madrid-6, Spain

21-22 Oct.

Upton, NY, USA

22nd Eastern Theoretical Physics Conference

L. Trueman, Physics Dept., Brookhaven National Lab. Upton, NY 11973

21-23 Oct.

Chester, UK

Annual Conf. of British Assoc. for Crystal Growth

F.W. Ainger, Plessey Research (Caswell) Ltd., Allen Clark Res. Centre, Caswell, Towcester, Northants. NN12 8EQ

21-26 Oct.

Messina, Italy

69 th Nat. Congress of Italian Physical Society

Ms. E. Mazzi, Società Italiana di Fisica, Via L. degli Andalò 2, I-40124 Bologna

Ab: 30.6 .83 / PP / 700 / Italian, Engl. / Lit. 10000 (mbrs. SIF); 15000 (others)

22-24 Oct.

6th Annual Conf. on Medical Physics

Srinagar, India

S. Kaul, 6th AMPI Conf., Sher-1-kashmir, Inst. of Medical Sciences, Srinagar 190011

23-26 Oct.

Geneva, Switzerland

9th European Conf. on Optical Communication

Conf. Secr. ECOC 83, A. Sollberger, Inst. for Applied Physics, Swiss Fed. Inst. of Technology, $\mathrm{CH}-8093$ Zurich

Ab: 30.4.83 / PP / URSI / incl. proc. SFr. 550. - before 15.9. : 590. - after

24-26 Oct.

Advanced Infrared Detectors and Systems London, UK IEE Conf. Dept., Savoy Place, London WC2R OBL $A b: 24.3 .83 / P: 29.7 .83$

27-28 Oct.

Stanford, CA, USA

10th Annual Stanford Synchrotron Rad. Lab. Users Group Meeting

B. Patterson, Stanford Univ., SSRL/SLAC Bin 69 , Stanford, CA 94035

31 Oct. -4 Nov.

Boston, MA, USA

1983 Symposium of American Vacuum Society

Exec. Secr., American Vacuum Soc.

335 E. 45th Str., New York, NY' 10017

2-4 Nov.

Bad Honnef, FRG

Collective Excitations in Nucle

P. von Brentano, Inst. f. Kernphysik d. Universität, Zülpicher Str. 77, D-5000 Köln 41

NP / $60 /$ inv.

6-11 Nov.

Pittsburgh, PA, USA

Magnetism and Magnetic Materials Conference

F.J. Werner, Westinghouse R. \& D. Center,

130 Beulah Rd. Pittsburgh, PA 15235

7-11 Nov.

Los Angeles, CA, USA

Meeting of Plasma Physics Division (APS)

The American Physical Society,

335 E. 45th Str., New York, NY 10017

7-11 Nov.

Trieste, Italy

Applications of Physics to Medicine and Biology

P. Baxa, ICTP, POB 586, 1-34100 Trieste

A: 20.9 .83 / Ab: 20.7 .83 / IUPAP

7-11 Nov.

Toulouse, France

Workshop: Problems of Collapse and Numerical Relativity

Monique Signore, DEMIRM, Observatoire de Meudon, F-92190 Meudon

10-16 Nov.

Düsseldorf, FRG

9th Int. Congress and Exhibition: Instrumentation and Automation (INTERKAMA 83)

INTERKAMA 83, NOWEA

Postfach 320203, D-4000 Düsseldorf 30

13-19 Nov.

Osaka, Japan

th Int Conf on Secondary lon Mass Spectrometry Osaka Univ, 1-1 Machikaneyama-cho, Toyonaka, Osaka 560

A: 31.7.83 / Ab: $30.4 .83 / \mathrm{PP}: 13.11 .83 /$ incl. proc. Y $30000 .-; 20000 .-$ students (before 31.7.): 


\section{European Physical Society}

Postal Address:

POB 69

CH-1213 Petit-Lancy 2

T. (22) $931130 / 32$

Tx. 423455 dema ch

Cables: Europhysics Genève

Location:

27. Chemin de la Vendée

Petit-Lancy, Geneva

Access:

Executive Secretary:

Chemin du Banc-Bénit

G. Thomas

\section{Past Presidents}

1968-1970:

G. Bernardini, Pisa

1970-1972:

E. Rudberg, Stockholm

1972-1976:

H.B.G. Casimir, Eindhoven 1976-1978:

I. Ursu, Bucharest
1978-1980:

A. Zichichi, Bologna

1980-1982:

A.R. Mackintosh, Copenhagen 1982-

J. Friedel, Orsay

\section{Honorary Members}

G. Bernardini, Pisa, Italy

H.B.G. Casimir, Heeze,

The Netherlands

P.L. Kapitza, Moscow, USSR

P.A.M. Dirac, Florida, USA

V.F. Weisskopf, Cambridge, USA

\section{Individual Ordinary Members}

In June 1983 the number of Individual Ordinary Members on the books of the Society totalled 3376 . The names of the nine delegates to Council who represent these mem. bers are given below.

\section{I.O.M. Delegates in Council}

Delegates are elected by a ballot of all the Individual Ordinary Members for a term of three years. The term is automatically extended if the delegate is elected to the Executive Committee.

$+K$. Bethge, Frankfurt/Main

+ J. Devreese, Antwerp

+ H. De Waard, Groningen

J. Ernest, Marcoussis

W. Heine, Cambridge

F. Janouch, Stockholm

+ J. Niederle, Prague

T. Riste, Kjeller

+ F. Fumi, Genoa

+ in last year of term

\section{Executive Committee}

President: J. Friedel

Laboratoire de Physique des Solides

Université Paris IX, Bâtiment 510

F-91405 Orsay

T. (6) $9416940 / 9285986$

Vice-President: G.H. Stafford

St. Cross College

Oxford OX1 3LZ, UK

T. (865) 512411

\section{Secretary: W.J. Merz \\ Laboratories RCA Ltd \\ Badenerstrasse 569 \\ $\mathrm{CH}-8048$ Zürich \\ T. (1) 526350}

Vice-Secretary: $\mathrm{H}$. de Waard

Labor, voor Algemene Natuurkunde

Westersingel 34

NL-9718 CM Groningen

T. (50) 115241

Treasurer: D. Kuhn

Institut für Experimentalphysik

Abteilung Hochenergiephysik

Sillgasse 8

A-6020 Innsbruck

T. $(5222) 724 / 6670$

Vice-Treasurer: G. Preparata

Via Vodice 27

1-00195 Rome

T. (80) 331044

\section{Auditors:}

G. Landwehr, Grenoble

P. Radvanyi, Gif-sur-Yvette

FIDES, Geneva
Members:

K.E. Ganzhorn

IBM Deutschland GmbH

Postfach 800880

D-7000 Stuttgart 80

T. (711) 7852501

J. Kaczer

Institute of Physics

Czechoslovak Academy of Sciences

$\mathrm{Na}$ Slovance 2

CS-180 40 Prague-Liben

T. (2) 842241

N. Kroo

Central Research Institute for Physics

POB 49

$\mathrm{H}-1525$ Budapest

T. (1) 166536

\section{O.V. Lounasmaa}

Low Temperature Laboratory

Helsinki University of Technology

SF-02150 Espoo 15

T. (0) 4566234

K.K. Rebane

Academy of Sciences

of the Estonian SSR

Kohtu 6

USSR-200103 Tallinn

T. 442129

\section{Member Organizations}

(X) $Y$

$X=$ No. of members in the Society
or Group

$\mathrm{Y}=$ No. of delegates to Council

Ampere Group (220) 1

Del. G.J. Béné

Pres. K.H. Hausser

Max-Planck Institut für Medizinische

Forschung, Jahnstrasse 29

D-6900 Heidelberg

Sec-Gen. G.J. Béné

Université de Genève, Section de

Physique, quai Ernest-Ansermet 24

$\mathrm{CH}-1211$ Geneva 4

T. (22) 219355

Austrian Physical Society (680) 2

Dels. G. Bauer

$$
\text { D. Kuhn }
$$

Pres. H. Paul

Institut für Experimentalphysik

Johannes Kepler Universität Linz

A-4040 Linz

Sec. H. Winter

Institut für Allgemeine Physik

TU Wien

Karlsplatz 13

A-1040 Wien

T. (222) $5601 / 3369$

Belgian Physical Society (400) 1
Del. J.M. Gilles
Pres. A. Deruytter
CBMN Steenweg op Retie
B-2440 Geel
Gen-Sec. P. Fettweis
CEN/SCK
Boeretang 200
B-2400 Mol
T. (14) 311801

Bulgarian Acad. of Sciences (105)

Del. P. Simova

Pres. A. Balevski

Sec-Gen. G. Bliznakov

Foreign Relations Department

7 November str. No. 1

Sofia BG

T. 877731

Danish Physical Society (331) 1

Del. J. Als-Nielsen

Pres. P.G. Hansen

Sec. J. Bøttiger

Institute of Physics

University of Aarhus

DK-8000 Aarhus C

T. (6) 128899
Dept. of Gen. Physics and Astronomy, USSR Acad. of Sciences

(2000) 4

Dels. Y.V. Gulaev V.S. Letokho K.K. Rebane

Sec-Gen. A.M. Prokhorov

Sci-Sec. S. Markyanov

USSR Academy of Sciences

Leninskyi Prospekt 14

USSR-Moscow B-71

T. 2322910

Eötvős Lorand Physical Society (625) 2

Dels. I. Abonyi

N. Kroo

Pres, G.J. Csikai

Institute of Experimental Physics

Kossuth University, Bem tér $18 /$

H.4026 Debrecen

Sec-Gen. N. Kroo

Eötvös Lorand Fisikai Tarsulat

Anker köz 1, POB 240

$\mathrm{H}-1368$ Budapest

T. (1) 227040
Finnish Physical Society (772) 2 Dels. R. Hämäläinen O.V. Lounasmaa

Pres. H.I. Miettinen

Research Inst. for Theoretical Physics

University of Helsinki

Siltavuorenpenger $20 \mathrm{C}$

SF-00170 Helsinki 17

Sec. O. Lehtelä

Department of Physics

University of Helsinki

Siltavuorenpenger $20 \mathrm{D}$

SF-00170 Helsinki 17

T. (0) 650211

French Physical Society (2200) 4

Dels. J. Friedel

$$
\begin{aligned}
& \text { F. Netter } \\
& \text { Y. Quere }
\end{aligned}
$$$$
\text { P. Radvanyi }
$$

Pres. C. Manus

CEN-Saclay DPh-G-SPAS

Boite Postale 2

F-91191 Gif-sur-Yvette

Sec-Gen. M. Barrère

Société Francaise de Physique

33 rue Croulebarbe

F-75013 Paris

T. (1) 7073298 


\section{Member Organizations} Contd.

German Physical Society (7400) 5 Dels. O.G. Folberth K.E. Ganzhorn

G. Grieger

G. Landwehr

J. Treusch

Pres. K.J. Schmidt-Tiedemann

Philips GmbH

Vogt-Kölln-Strasse 30

D-2000 Hamburg 54

Gen-Sec. W. Heinicke

Deutsche Physikalische Gesellschaft

Hauptstrasse 5

D-5340 Bad Honnef

T. (2224) 71061

Hellenic Physical Society (50) 1

Pres. N.G. Antoniou

Hellenic Physical Society

6 Griveon Street

GR-Athens 144

T. (1) 635701

Icelandic Physical Society (45)

Del.

Pres. H.Kr. Gudmundsson

Science Institute, University of Iceland

Dunhaga 3

IS-107 Reykjavik

Sec. S. Sigurosson

Karsnesbraut 18

IS-200 Kopavogi

Institute Ruder Boskovic (100)

Del. N. Zovko

Dir-Gen. V. Kundic

Sec. N. Zovko

Institute Ruder Boskovic

Bijenicka 54, POB 1016

YU-41001 Zagreb

T. (41) 424093

Institute of Physics (13500) 5

Dels. Sir Robert Clayton

L. Cohen

G.H. Stafford

B.L.H. Wilson

Pres. Sir Robert Clayton

The General Electric Co. Ltd.

Hirst Research Centre, East Lane

Wembley, Middlesex HA9 7PP UK

Exec-Sec. L. Cohen

The Institute of Physics

47 Belgrave Square

London SW1X $80 \times$ UK

T. (1) $2356111 \mathrm{Tx} .918453$
Israel Physical Society (240) 1

Del. M. Carmeli

Pres. M. Carmeli

Sec. A. Gersten

Department of Physics

Ben-Gurion University

Beer-Sheva 84-120, Israe

T. (57) 64274

Italian Physical Society (1023) 3

Dels. G. Preparata

$$
\text { R.A. Ricci }
$$

$$
\text { A. Rubbino }
$$

Pres. R.A. Ricci

Istituto di Fisica

Via Marzolo 8

1-35100 Padova

Sec. E. Mazzi

Società Italiana di Fisica

Via L. degli Andalo 2

1-40124 Bologna

T. (51) 331554

Netherlands' Physical Society

(2851) 4

Dels. J.B. Dragt

J.J.J. Kokkedee

E.W.A. Lingeman

A. van der Woude

Pres, J.J.J. Kokkedee

Afdeling Technische Natuurkunde

Technische Hogeschool te Delft

Lorentzweg 1, POB 5046

NL-2600 GA Delft

Sec. F.R. de Boer

Nederlandse Natuurkunde Vereniging

Princetonplein 5, РOB 80.000

NL-3508 TA Utrech

T. (30) 532329

Norwegian Physical Society (608) 2

Dels. T. Engeland

$$
\text { R.S. Sigmond }
$$

Pres. E. Osnes

Department of Physics

University of Oslo

$\mathrm{N}$-Blindern, Oslo 3

Sec. G. Jarrett

Institutt for Atomenergi, РОВ 40

N-2007 Kjelle

T. (2) $712560 \mathrm{Tx} .16361$ atom n

Phys. Sect. Union of Czechoslovak

Mathematicians and Physicists (500) 2

Dels. J. Kaczer

P. Mrafko

Pres. J. Kaczer

Sec. J. Fousek

Institute of Physics

Czechoslovak Academy of Sciences

Na Slovance 2

CS-18040 Prague 8

T. (422) 842241 Tx. 122018 atom c
Physical Society of the German

Democratic Republic (1529) 3

Dels. D. Hommel

U. Krecker

K. Thiessen

Pres. R. Rompe

Sci-Sec. R. Linke

Physikalische Gesellschaft de

Deutschen Demokratischen Republik

Am Kupfergraben 7

DDR-1080 Berlin

T. (2) 2000691

Physical Sect. Union of Yugoslav Societies of Mathematicians,

Physicists and Astronomers (200) 1

Del. A. Milojevic

Pres. V. Dajovic

Gospodar Jevremova 45

YU-11000 Belgrade

Sec-Gen. P. Obradovic

Institute of Mathematics and Physics

University of Titograd, Cetinjski put b.b.

YU-81000 Titograd

T. (81) 52224

Polish Physical Society (1600) 3

Dels. T. Skalinski$$
\begin{aligned}
& \text { E. Skrzypczak } \\
& \text { W. Zych }
\end{aligned}
$$

Pres. T. Skalinski

Institute of Physics of the

Polish Academy of Sciences

Al. Lotnikow $32 / 46$

PL-02-668 Warsaw

Gen-Sec. W. Zych

Polskie Towarzystwo Fizyezne

Hoza 69

PL-00-681 Warsaw

T. (22) 212668

Portuguese Physical Society (200) 1

Del. A.F. Martins

Sec-Gen. A.F. Martins

Sec Ext Rel. J. Dias de Deus

Sociedade Portuguesa de Fisica

CFMC-INIC

Av. Prof. Gama Pinto 2

P. 1699 Lisbon Codex

T. (1) 773338

Romanian National Committee

for Physics (300)

Del. A. Corciovel

Pres. I. Ursu

National Council for

Science and Technology

Bucharest R

Sec. A. Corciove

Institute for Atomic Physics

POB 5206

Bucharest $R$

T. $807040 \mathrm{Tx} .11397$ fiatom r
Royal Irish Academy (60) 1

Del. A. Thompson

Pres. T.P.G. McGreevy

Sec. A. Thompson

Irish National Committee for Physics

19 Dawson Street

Dublin 2 IRL

T. (31) 762570

Royal Spanish Society of Physics

(200) 1

Del. C. Sanchez del Rio

Pres. C. Sanchez del Rio

Sec-Gen. A. Tiemblo

Real Sociedad Española de Fisica

Facultades de Ciencias

Ciudad Universitaria

Madrid-3 E

T. (1) 2433879

Swedish Physical Society (935) 2

Dels. R. Hellborg

B.E.Y. Svensson

Pres. B.E.Y. Svensson

Institute of Theoretical Physics

University of Lund

Sölvegatan 14

S-223 62 Lund

Sec. R. Hellborg

Institute of Physics

University of Lund

Sölvegatan 14

S-22362 Lund

T. (46) 107644

Swiss Physical Society (1190) 3

Dels. J.P. Blaser

$$
\text { J. Kern }
$$

W.J. Merz

Pres. J. Kern

Sec. B. Vaucher

Institut de Physique

University of Fribourg

Pérolles

$\mathrm{CH}-1700$ Fribourg

T. (37) 826230

Turkish Physical Society (302) 1

Del. E. Inönü

Pres. E. Inönü

Sec. M. Serdaroglu

Fizik Bölümû

Bogaziçi Universitesi, P.K. 2

Bebek - Istanbul TR

T. (11) 653400

Collaborating Society

American Physical Society

335 East 45 th St.

New York

N.Y. 10017 USA

T. (212) 6827341

\section{EPS Advisory Committees}

\section{Conference Committee}

Chair. S. Methfesse

Sec. K. Westerholt

Institut für Experimentalphysik

Ruhr Universität

Postfach 2148

D-4630 Bochum

T. (234) $7003649 / 50$

O. Akyuz, Bogaziçi Univ., Istanbul

F. Bassani, High School, Pisa

B. Cagnac, Univ. Pierre et Marie Curie,

Paris

I.A. Dorobantu, Central Institute of Physics, Bucharest

F.A. Gianturco, Institute of Chemical

Physics, Rome

G. Grieger, MPI for Plasma Physics, Garching

J. Heijn, NNN Physics Laboratory, Utrecht

1. Iori, Institute of Physics, Milan

A. Kujawski, Polish Academy of Sciences, Warsaw

A. Linek, Czechoslovak Academy of Sciences, Prague

A. Lösche, University, Leipzig

1. Lovas, Central Research Institute for Physics, Budapest

N. Lund, Danish Space Research Inst. Lyngby

D. Lundquist, Bromm

W.J. Merz, RCA Laboratories, Zürich

F. Mueller, University, Nijmegen

W.S. Newman, CERN, Geneva

J.S. Nilsson, Institute of Theoretical

Physics, Göteborg

S. Popovic, Rudjer Boskovic Inst. Zagreb

P. Radvanyi, National Laboratory

Saturne, Gif-sur-Yvette

J. Smidt, Laboratory for Technical Physics, Delft

S.D. Smith, Heriot Watt University, Edinburgh

J.-P. Swings, Institute of Astrophysics, Cointe-Liège

G. Thomas, EPS Secretariat

L. Van Gerven, University, Leuven

D. Wegener, University, Dortmund 


\section{Applied Physics and Physics in Industry}

Chair. G. Pfister

Cerberus AG

CH-8708 Männedorf

T. (1) 9226263

N. Ben-Yoseph, Hebrew University, Jerusalem

B. Bölger, Philips' Research Lab. Eindhoven

J. Ernest, CGE Corporate Research Centre, Marcoussis

A. Ferro-Milone, National Electrotechnical Institute, Turin

J. Gyulai, Central Research Institute for Physics, Budapest

F. Lappe, Hoechst AG, Frankfurt

D. Lundqvist, Bromma

A. Olès, Academy of Mining and

Metallurgy, Cracow

C.P. O'Toole, Royal Irish Acad., Dublin

S. Popovic, Institute of Physics, Belgrade

G. Raoult, Lab. of Radioelectricity \& Solid State, Aubière

F. Scarlat, Central Institute of Physics, Bucharest

A. Sokollu, Marmara Research Institute, Istanbul

K. Volenik, State Research Institute, Prague

B.L.H. Wilson, Plessey Ltd., Towcester

G. Winstel, Siemens AG, Munich

R. Zelazny, Institute for Nuclear

Research, Swierk k. Otwocka

\section{Finance Committee}

Chair.: P. Toennies

Max-Planck-Institut für Strömungs-

forschung

Postfach 867

D-3400 Göttingen

T. (551) $7092600 / 2601$

Tx. 09-6768 mpstr d

J. Kaczer, Czechoslovak Academy of Sciences, Prague

D. Kuhn, Institute of Experimental

Physics, Innsbruck

A. Messiah, Institute of Fundamental

Research, Paris

G. Preparata, Institute of Physics, Bari

G. Thomas, EPS Secretaria

L.A. Thomas, GEC, Wembley

\section{Physics and Society}

Chair. D.H. Parkinson

South Bank

47 Abbey Road

Malvern, WR14 $3 \mathrm{HH}$ UK

T. (6845) 5423

Sec. K. Schultze

Physikalisches Institut III

RWTH Aachen

Physikzentrum, Sommerfeldstrasse

D-5100 Aachen

T. (241) 807330

G.J. Béné, University, Geneva

I. Brandus, Central Institute of Physics,

Bucharest

G.J. Csikai, University, Debrecen

M.J. Higatsberger, University, Vienna

G. Landwehr, CNRS, Grenoble

C. le Pair, FOM, Utrecht

E. Lillethun, University, Bergen

O. Piller, Federal Office for Meteo-

rology, Bern

I. Slaus, Rudjer Boskovic Inst., Zagreb

G.A. Smolensky, A.F. Ioffe Physico

Technical Institute, Leningrad

I. Stoll, Technical Institute, Prague

M. Tomak, M.E.T. Univ., Ankara

W. Turkenburg, University, Utrecht

H. van Regemorter, Observatory,

Meudon

J. Vlachy, Prague

W. Zych, Technical University, Warsaw

\section{Scientific Freedom}

Chair.: J.M. Charap

Queen Mary College

Mile End Road

London E1 4NS UK

T. (1) 9804811

A. Guinier, Univ, Paris-Sud, Orsay

F. James, CERN, Geneva

E.T. Karlsson, Inst. of Technology, Uppsala

\section{Publications Committee}

Chair. G. Eilenberge

Institut für Festkörperforschung der Kernforschungsanlage Jülich GmbH Postfach 1913

D-5170 Jülich 1

T. (2461) 613136

Sec. E.N. Shaw

EPS, POB 69

CH-1213 Petit-Lancy 2

T. (22) 931130

G. Alaga, University, Zagreb

K. Appert, EPFL, Lausanne

J.M. Araujo, Faculty of Sciences, Oporto

M. Balkanski, Univ. Pierre et Marie

Curie, Paris

D. Barb, Central Institute of Physics, Bucharest

G. Bellini, Institute of Physical Sciences, Milan

A.B. Berezin, A.F. loffe PhysicoTechnical Institute, Leningrad

P.S.H. Bolman, North-Holland Publ. Co., Amsterdam

J. Campos, University, Madrid

N. Cindro, Rudjer Boskovic Inst. Zagreb

B.R. Coles, Taylor \& Francis, London

H. Demiryont, Faculty of Physics, Istanbul

J. Devreese, University, Antwerp

A. Gibson, JET, Abingdon

J. Kaluzny, Pedagogical Faculty, Trnava

$H$. Lotsch, Springer-Verlag, Heidelberg

M.R.C. McDowell, Royal Holloway

College, Egham

F. Mezei, ILL Grenoble

L. Michel, IHES, Bures-sur-Yvette

J.W. Miltenburg, Philips, Eindhove

H. Mitter, University, Graz

F. Netter, CEN-Saclay, Gif-sur-Yvette

E.R. Pike, Royal Radar Establishment, Great Malvern

G. Preparata, Rome

E. Preuss, MPI for Radioastronomy Bonn

G. Raoult, Lab, of Radioelectricity \& Solid State, Aubière

W. Rittberger, FIZ Energy, Physics \& Maths., Eggenstein-Leopoldshafen

G. Tibell, Gustaf Werners Institute, Uppsala

L. Van Gerven, University, Leuven

H.P. Weber, University, Bern

\section{Europhysics News}

Chair. J. Muller

Section de Physique,

Université de Genève,

Quai Ernest-Ansermet 24

$\mathrm{CH}-1211$ Geneva 4

T. (22) 219355

Sec. E.N, Shaw

EPS, POB 69

$\mathrm{CH}-1213$ Petit-Lancy 2

T. (22) 931130

K. Appert, EPFL, Lausanne

J. Auth, DDR National Committee

for Physics, Berlin

-A. Baratoff, IBM Res. Lab., Rüschlikon

- G.J. Béné, University, Geneva

A.B. Berezin, A.F. Ioffe Physico

Technical Institute, Leningrad

E. Burzo, Central Institute of Physics, Bucharest

J. Campos, University, Madrid

J. Charap, Queen Mary College, London

E. Dreisigacker, Physik-Verlag,

Weinheim

M. Erbudak, ETH, Zürich

P. Erdös, University, Lausanne

S. Focardi, Inst. of Physics, Bologna

F.J. Kevdes, Kossuth University,

Debrecen

- G.R. Macleod, CERN, Geneva

- A. Maeder, Geneva Observatory, Versoix

W.S. Newman, CERN, Geneva

J. Niederle, Czechoslovak Academy of Sciences, Prague

C.I. Pedersen, Inst. of Physics, Bristol

J. Sharpey-Schafer, University,

Liverpool

A. Sobiczewski, University, Warsaw

M. Taieb, CENG, Grenoble

G. Thomas, EPS Secretariat

F. Troyon, Plasma Physics Research

Centre, Lausanne

N. Urli, Ruder Boskovic Inst., Zagreb

$\mathrm{H}$. van Krugten, University, Delft

- $=$ Member of Editorial Board

\section{EPS Divisions}

\section{Astronomy and Astrophysics Division (335)}

Chair. C. Cesarsky

Service Electronique Physique

CEN-Saclay, POB 2

F-91191 Gif-sur-Yvette

T. (6) 9084344

Sec. J.-P. Swings

Institut d'Astrophysique

Université de Liège

B-4200 Cointe-Liège

T. (41) 529980

Board Members:

C. Chiuderi, University, Florence

J. Kota, Central Research Institute

for Physics, Budapest

N. Lund, Danish Space Research Institute, Lyngby

H. Ögelman, Middle East Technica

University, Ankara

K. Pounds, University, Leicester

E. Preuss, Max-Planck Institute for

Radioastronomy, Bonn

M. Tarenghi, ESO, Garching

J. Trümper, Max-Planck Institute for

Extraterrestrial Physics, Garching

J. van Paradijs, Astronomical Institute

Amsterdam

G.T. Zatsepin, Lebedev Institute,

Moscow
Solar Physics Section (107)

Chair. M. Kuperus

Sterrewacht Sonnenborgh

Zonnenburg 2

NL-3512 NL Utrecht

T. (30) 312841

Sec. R.W.P. McWhirther

Rutherford Appleton Laboratory

Chilton, Didcot

Oxon OX $1100 \times$ UK

T. (235) 21900

Board Members:

C. Chiuderi, University, Florence

C.J. Durrant, University, Sidney

J. Leroy, Observatory,

Bagnères de Bigorre

P. Maltby, University, Oslo

H. Nussbaumer, ETH, Zürich

M.A. Pick, Observatory, Meudon

R.J. Rutten, Observatory, Utrech

E.H. Schröter, Institute for Solar

Physics, Freiburg

J.O. Stenflo, ETH, Zürich

N. Weiss, University, Cambridge

\section{Atomic and Molecular Physics Division (633)}

Chair. F.A. Gianturco

Institute of Chemical Physics

University of Rome

Piazzale delle Scienze 5

1.00185 Rome

T. (6) 4991

Sec. T. Andersen

Institute of Physics

University of Aarhus

DK-8000 Aarhus C

T. (6) 128899

Board Members:

H. Erhardt, University, Kaiserslautern

H. B. Gilbody, University, Belfast

I. Hertel, University, Berlin (W)

W. Kolos, University, Warsaw

J.C. Lehmann, University VI, Paris

J. Los, FOM, Amsterdam

M.R.C. McDowell, Royal Holloway College, Egham

D.N. Stacey, University, Oxford

F. Wuilleumier, University Paris-Sud, Orsay

The Chairmen and Secretaries of the Sections serve also on the Divisional 
Atomic \& Molecular

Phys. Div. Contd.

\section{Chemical Physical Section (247)}

Chair. E.A.C. Lucken

Département de Chimie Physique

Sciences II, 30 quai E. Ansermet

$\mathrm{CH}-1211$ Geneva 4

T. (22) 219355

Sec. R.E. Bühler

Physical Chemical Laboratory

ETH, Universitätstrasse 22

CH-8092 Zurich

T. (1) 2562211

\section{Board Members:}

R. Daudel, Applied Wave

Mechanics Centre, Paris

R. Gaspar, Kossuth Univ., Debrecen

L. Jansen, Battelle Institute, Geneva

E. Roth, CEN-Saclay, Gif-sur-Yvette

J.P. Toennies, MPI for Fluid Mechanics, Göttingen

A. van der Avoird, University, Nijmegen

G. Wagnière, University, Zurich

D.A. Young, Chemical Soc., London

\section{Electronic and Atomic}

Collisions Section (338)

Chair. F. Linder

Universität Kaiserslautern

Erwin-Schrödinger-Strasse

D-6750 Kaiserslautern

T. (631) 8542383

Sec. A. Giardini-Guidoni

CNEN Laboratorio Spettroscopia

Molecolare, POB 65,

1-000 44 Frascati

T. (6) $942 \quad 1041$

\section{Board Members:}

J.-P. Gauyacq, Univ. Paris-Sud, Orsay H.G.M. Heideman, RU, Utrecht C.J. Joachain, Free Univ., Brussels M. Kurepa, Inst. of Physics, Belgrade W. Lindinger, University, Innsbruck V. Martisovits, University, Bratislava M.R.C. McDowell, Royal Holloway College, Egham

K. Taulbjerg, University, Aarhus

L. Wolniewicz, University, Torun

\section{Molecular Physics Section} (326)

Chair. S. Leach

Laboratoire de Photophysique Moléculaire

Bâtiment 213, Université de Paris-Sud

F-91 405 Orsay Cedex

T. (6) 9417909

Sec. I. Dubois

Institut d'Astrophysique

Université de Liège

B-4000 Cointe-Liège

T. (41) 529980 Tx. 41264 astr lg/d

Board Members:

S. Califano, University, Florence

R.M.N. Dixon, University, Bristol

P. Erman, Royal Institute of Technology,

Stockholm

B. Jezowska, University, Wroclaw

I. Kovacs, Polytechnic, Budapest

V.S. Letokhov, Academy of Sciences, Moscow

I.M. Mills, University, Reading

Z. Pajak, University, Poznan

S.D. Peyerimhof, Unniversity, Bonn

S. Stolte, University, Nijmegen

M. Winnewisser, University, Giessen

C. Wolf, University, Stuttgart

B. Zuev, Academy of Sciences, Tomsk

\section{Condensed Matter Division (1090)}

Chair. J. Devreese

Department of Physics

University of Antwerp (UIA)

Universiteitsplein 1

B-2610 (Wilrik) Antwerp

T. (31) 305980

Sec. V. Van Doren

UIA Department of Physics

Universiteitsplein 1

B-2610 (Wilrijk) Antwerp

T. (31) $305980 / 317$

Board Members :

A. Abrikosov, USSR Academy of

Sciences, Moscow

H. Bilz, MPI for Solid State Research, Stuttgart

R.M.J. Cotterill, Technical Univ.,

Lyngby

B. Feuerbacher, DFVLR, Cologne

$H$. Grimmeiss, Inst. of Technology,

Lund

G. Harbeke, RCA, Zurich

V. Heine, Cavendish Lab., Cambridge

W. Holzapfel, University, Paderborn

G.M. Kalvius, Technical Univ., Munich

H.H. Kausch, EPFL, Lausanne

S.O. Lundqvist, Institute of Theoretical Physics, Göteborg

E. Mooser, EPFL, Lausanne

F.M. Mueller, University, Nijmegen

D. Thoulouze, CRTB - CNRS, Grenoble

C. Rizzuto, University, Genoa

M. Weger, Hebrew University, Jerusalem

G. Winstel, Siemens AG, Munich

A. Zawadowski, University of

California, Los Angeles

\section{Low Temperature Section (322)}

Chair. C. Rizzuto

stituto di Scienze Fisiche

Università, Viale Benedetto XV, 5

1-16132 Genoa

T. (10) 515055

Committee Members

Y. Bruynseraede, University, Leuven

G. Deutscher, University, Tel-Aviv

1. Kirchner, Lab. for Low Temperature

Physics, Budapest

M. Krusius, University, Turku

F. Pobell, KFA, Jülich

H. Postma, University, Delft

F.B. Rasmussen, H.C. Orsted Inst. Copenhagen

L. Rinderer, University, Lausanne

D. Thoulouze, CRTB-CNRS, Grenoble

W.F. Vinen, University, Birmingham

\section{Magnetism Section (424)}

Chair. G.M. Kalvius

Technische Universität

Arcisstrasse 21

D-8000 Munich 2

T. (98) 32092501

Committee Members:

W.D. Corner, University, Durham

L.J. de Jongh, Kammerlingh Onnes

Laboratory, Leiden

R. Elliott, University, Oxford

P. Erdös, University, Lausanne

W. Haubenreiser, University, Jen

A. Hubert, University, Erlangen

V. Kambersky, Academy of Sciences. Prague

R. Pauthenet, ILL, Grenoble

S. Shtrikman, Weizmann Institute,

Rehovot

V. Smirnov, A.F. loffe Physico

Technical Institute, Leningrad

W. Suski, Polish Academy of

Sciences, Wroclaw

\section{Macromolecular Physics Section} (196)

Chair. H.H. Kausch

Polymer Laboratory

Ecole Polytechnique Fédérale

$32 \mathrm{Ch}$. de Bellerive

$\mathrm{CH}-1007$ Lausanne

T. (21) 472841

Committee Members:

F.J. Baltà-Calleja, Institute of

Chemical Physics, Madrid

G. Bodor, Polymer Research Institute, Budapest

A. Keller, University, Bristo

M. Kryszewski, Polish Academy of

Sciences, Lodz

J.P. Mercier, High Polymer Lab.

Louvain-la-Neuve

L. Monnerie, ESPCI, Paris

B. Ronby, Royal Institute of

Technology, Stockholm

H. - K. Roth, Karl-Marx University,

Leipzig

W. Ruland, Philipps Univ., Marburg

G.L. Slonimsky, USSR Academy of

Sciences, Moscow

J. Spevacek, Inst. of Macromolecular

Chemistry, CVAS, Prague

L.C.E. Struik, TNO, Delft

G. Zerbi, University, Trieste

\section{Metals Section (473)}

Chair. R.M.J. Cotterill

Dept. of Structural Properties of

Materials, Technical University

DK-2900 Lyngby

T. (2) 882222

Committee Members:

D. Buschow, Philips' Research Labs. Eindhoven

G. Caglioti, Institute of Nuclear

Engineering, Milan

D.J. Fabian, Strathclyde University, Glasgow

O. Fischer, University, Geneva

J.P. Gaspard, University, Liege

Semiconductors and Insulators (557)

Chair. G. Harbeke

RCA Laboratories Ltd

Badernerstrasse 569

$\mathrm{CH}-8048$ Zurich

T. (1) 526350

Committee Members

F.W. Ainger, Plessey Ltd, Towcester

D. Bäuerle, University, Linz

F. Beleznay, Institute for Technical

Physics, Budapest

R. Blinc, Josef Stefan Inst., Ljublijana

A. Frova, University, Rome

H. Grimmeiss, University, Lund

D. Kaplan, Thomson-C.S.F., Orsay

S. Porowski, Acad. of Sciences,

Warsaw

Surfaces and Interfaces (357)

Chair. B. Feuerbacher

Institut für Raumsimulation

DFVLR - Linder Höhe

D-5000 Köln 90

T. (2203) 6012176

Committee Members:

G. Benedek, Inst. of Physics, Milan

A. Cassuto, LARIGS, Nancy

G Ertl, University, Munich

N. Garcia, University, Madrid

B. Joyce, Philips' Research Lab.

Redhill

B.I. Lundqvist, Chalmers Univ., Göteborg

A. van Oostrom, Philips' Research

Labs., Eindhoven

B. Velicky, Institute of Physics, CVAS, Prague

R.F. Willis, University, Cambridge

K.F. Wojciechowski, University,

Wroclaw

\section{Computational Physics Group (349)}

Chair. G. Diercksen

Max-Planck-Institut für Astrophysik

Fuhringer Ring 6

D-8000 München 40

T. (89) 32990

Sec. P. Van Binst

University of Brussels II HE

Bd. du Triomphe, POB 230

B-1050 Brussels

T. (2) 6413211

Board Members :

R.K. Böck, CERN, Geneva

L. Fischer, Comenius University, Bratislava

F.D. Gault, University, Durham

R. Gruber, EPFL, Lausanne

J.-P. Hansen, University VI, Paris

J. Linderberg, Dept. of Chemistry, University, Aarhus

J. Nadrchal, Institute of Physics,

CVAS, Prague

R.S. Peckover, Culcheth Laboratory, Warrington

B.P. Veltman, University of

Technology, Delft

\section{High Energy and Particle} Physics Division (530)

Chair. J.M. Charap

Department of Physics

Queen Mary College, Mile End Ro.

London E1 4NS UK

T. (1) 9804811

Sec. W. Bartel

DESY

Notkestrasse 85

D-2000 Hamburg 52 


\section{Nuclear Physics \\ Division (541)}

Chair. P. von Brentano Institut für Kernphysik der Universität Köln Zülpicher Strasse 77 D-5000 Köln 41 (Sülz) T. (221) $4703456 / 2796$

Sec. J. Sharpey-Schafer Oliver Lodge Laboratory University of Liverpool, POB 147 Liverpool L69 3 BX UK T. (51) 7096022

Vice-Chair. D. Berenyi Institute of Nuclear Research, Debrecen

\section{Board Members:}

A. Berinde, Central Institute of Physics, Bucharest

M. Bouten, CEN/SCK, Mol

D. Branford, University, Edinburgh

A. Budzanowski, Institute of Nuclear

Physics, Cracow

G.A.P. Englebertink, RU, Utrecht I. Iori, Institute of Physics, Milan J. Kantele, Physics Dept., Jyvăskylä K.P. Lieb, University, Göttingen V.S. Soloviev, JINR, Dubna G. Tibell, Gustaf Werners Inst. Uppsala

I. Ulehla, Charles University, Prague N. Vinh Mau, Univ. Paris-Sud, Orsay

G. Vourvopoulos, NRC Demokritos, Athens

H. Zingl, University, Graz

\section{Plasma Physics Division (302)}

\section{Quantum Electronics Division (274)}

Chair. A. Gibson

JET Joint Undertaking

T. (40) 742179

Sec. H. Welling

Institut für Angewandte Physik der Technischen Universität

Welfengarten 1

D-3000 Hannover

Centre de Recherches en Physique des Plasmas, 21 Ave. des Bains

$\mathrm{CH}-1007$ Lausanne

T. (21) 471111

T. (511) 7622452

Board Members :

F.R. Ausenegg, University, Graz

M.J. Colles, Heriot-Watt University, Edinburgh

Board Members:
R. Behrisch, MPI for Plasmaphysics, Garching

$H$. de Kluiver, FOM, Nieuwegein

G. Grieger, MPI for Plasmaphysics, Garching

J. Jacquinot, CEN, Fontenay-aux-Roses

R.V. Sagdeev, USSR Academy of Sciences, Moscow

M. Samain, CEN, Fontenay-aux-Roses

D.C. Schram, University of

Technology, Eindhoven

D. Sweetman, Culham Laboratory, Abingdon

R. Wienecke, Institut for Plasma

Research, Stuttgart

H. Wilhelmsson, Chalmers University, Göteborg

\section{Membership of Boards and Committees}

Membership of the EPS scientific Divisions (including the Sections) is restric ted to Individual Members of the Society. No limit is placed on the num ber of Divisions or Sections an Individual Member may join but adherence to at least one is expected. Details of the categories of Individual Members are given on page 10 and an application form for membership is presented on pages 11 and 12.

Boards and governing Committees of the Divisions and Sections are elected from amongst the Division or Section members although Member Organiza tions may present non-members as candidates. Those elected are expected to become Individual Members.

Elections are held every three years, a limit of six years continuous service being the maximum. In addition to the elected members, other members may be coopted to serve on the various Boards and Committees. The lists presented in this Directory reflect the position as of 1 June 1983. Changes will be reported in Europhysics News as they occur.
L'Air Liquide, Jouy en Josas, France

Battelle Geneva Research Centre,

Carouge-Geneva, Switzerland

BESSY - Berliner Elektronenspeicherring Ges.

für Synchrotronstrahlung, Berlin West

British Telecom Research Laboratories,

Martlesham Heath, England

Brown-Boveri \& Co. Ltd.

Baden, Switzerland

CEA - Commissariat à l'Energie Atomique,

Paris, France

CEGB - Central Electricity Generating Board,

London, England

Cerberus Ltd., Männedorf, Switzerland

CERN - European Organization for Nuclear Research, Gèneva, Switzerland

CGE - Cie Générale d'Electricité,

Paris, France

CNES - Centre National d'Etudes Spatiales,

Paris, France

CNET - Centre National d'Etudes des

Télécommunications, Lannion, France

CNR - Consiglio Nazionale delle Ricerche,

Rome, Italy

CNRS - Centre National de la Recherche

Scientifique, Paris, France

DESY - Deutsches Elektronen-Synchrotron, Hamburg, FRG

ECN - Netherlands Energy Research

Foundation, Petten, the Netherlands

EDF - Electricité de France, Paris, France

Elettronica San Giorgio - ELSAG SpA, Genoa Sestri, Italy

ELF - Société Nationale ELF Aquitaine, Paris, France

ENEA - Comitato Nazionale per la Ricerca e Sviluppo dell'Energia Nucleare e delle Energie Alternative, Rome, Italy

\section{Associate Members}

ESA - European Space Agency, Paris, France

$\mathrm{FIZ}$ Energie, Physik, Mathematik GmbH,

Eggenstein-Leopoldshafen, FRG

FOA - National Defence Research Institute,

Stockholm, Sweden

FOM - Foundation for Fundamental

Research on Matter, Utrecht, NL

General Electric Co. Hirst Research Centre,

Wembley, England

GSI - Gesellschaft für Schwerionenfor-

schung, Darmstadt, Fed. Rep. of Germany

Hahn-Meitner-Institut für Kernforschung,

Berlin-West

IBM Europe, Paris, France

ILL - Institut Max von Laue-Paul Langevin, Grenoble, France

INFN - Istituto Nazionale di Fisica Nucleare, Rome, Italy

JET Joint Undertaking, Abingdon, England

N.V. Kema, Arnhem, the Netherlands

Kernforschungszentrum Karlsruhe $\mathrm{GmbH}$ Karlsruhe, Fed. Rep. of Germany

KFA - Kernforschungsanlage Jülich, Jülich, Fed. Rep. of Germany

Matra, Saint-Quentin-en-Yvelines, France

Max-Planck-Institut für Festkörperforschung, Stuttgart, Fed. Rep. of Germany

Max-Planck-Institut für Plasmaphysik, Garching, Fed. Rep. of Germany

NORDITA - Nordisk Institut for Teoretisk Atomfysik, Copenhagen, Denmark

North-Holland Publishing Co. .

Amsterdam, the Netherlands

Oldelft, Delft, the Netherlands

ONERA - Office National d'Etudes et

Recherche Aérospatiale, Chatillon, France
Österreichisches Forschungszentrum

Seibersdorf $\mathrm{GmbH}$, Vienna, Austria

Pechiney-Ugine-Kuhlmann, Paris, France

Compagnie Française Philips,

Limeil-Brévannes, France

N.V. Philips' Gloeilampenfabrieken,

Eindhoven, the Netherlands

Physikalisch-Technische Bundesanstalt, Braunschweig, Fed. Rep. of Germany

Plessey Research (Caswell) Ltd.

Towcester, England

Laboratories RCA Ltd., Zürich, Switzerland

Renault, Rueil-Malmaison, France

Rhône-Poulenc, Courbevoie, France

Ris $\varnothing$ National Laboratory, Roskilde, Denmark

Sté. Etudes et Productions Schlumberger.

Clamart, France

Cie. de Saint-Gobain

Pont-à-Mousson, France

Siemens AG, Berlin \& Munich, FRG

SIN - Swiss Institute for Nuclear Research, Villigen, Switzerland

SPPIF - Soc. de Publication de Périodiques Internationaux et Français Solides, Masson, Paris, France

Springer-Verlag GmbH \& Co. KG, Heidelberg, Fed. Rep. of Germany

Standard Telecommunication Laboratories, Harlow, England

Taylor \& Francis Ltd., London, England

Thomson CSF, Paris, France

TNO - Netherland Organization for Applied Scientific Research, The Hague, NL

UK Atomic Energy Authority,

London, England

URANIT GmbH,

Jülich, Fed. Rep. of Germany 


\section{Europhysics Journals}

Journal

Acta Physica Polonica $A$ and $B$

Acustica

Advances in Physics

Astronomy and Astrophysics

Computer Physics Communications

Contemporary Physics

Czechoslovak Journal of Physics

Journal de Physique

Journal of Magnetism and Magnetic Materials

Journal of Optics

Journal of Physics A: Mathematical and General

Journal of Physics B: J. Phys. B Atomic and Molecular Physics

Journal of Physics C: J. Phys. C Solid State Physics

Journal of Physics D: J. Phys. D Applied Physics

Journal of Physics E: J. Phys. E Scientific

Instruments

Journal of Physics F: J. Phys. F Metal Physics

Journal of Physics G: J. Phys. G Nuclear Physics

Molecular Physics

Nuovo Cimento $A$

Optica Acta

Philosophical Magazine

Physica A

Physica $B+C$

Physica Scripta

Physics in Techno$\log y$

Plasma Physics

Reports on Progress in Physics

Revue de Physique Appliquée

Zeitschrift für Naturforschung

Zeitschrift für Physik A: Atoms and Nuclei

Zeitschrift für Physik B: Condensed Matter

Zeitschrift für Physik C: Particles $\&$ Fields

\section{Publisher}

Polish Academy of Sciences

S. Hirzel Verlag

Taylor \& Francis

Springer-Verlag

North Holland Pub. Co.

Taylor \& Francis of Sciences

Les Editions de Physique

North Holland Pub. Co.

S.P.P.I.F./Masson

The Institute of Physics

The Institute of Physics

The Institute of Physics

The Institute of Physics

The Institute of Physics

The Institute of Physics

The Institue of Physics

Molec. Phys.

Nuovo Cim. A

Opt. Acta

Phil. Mag.

Physica A

Physica $B+C$

Phys. Scr

Phys. Technol.

Plasma Phys.

Rep. Prog. Phys.

Revue Phys. Appl.

Z. Naturf

Z. Phys. A

Z. Phys. B

Z. Phys, C

Springer-Verlag

Taylor \& Francis

Italian Physical

Society

Taylor \& Francis

Taylor \& Francis

North-Holland Pub. Co.

North-Holland Pub. Co.

Royal Swedish Academy of Sciences

The Institute of Physics

Pergamon Press

The Institute of Physics

Editions de Phy sique

Verlag der Zeitschrift für Naturforschung

Springer-Verlag

Springer-Verlag
Czechoslovak Acad

\section{Individual Membership}

Make the European community of physicists stronger by becoming an Individual Member of the European Physical Society.

There are three categories of Individual Membership according to the Constitution of the EPS:

4a) Physicists who have contributed to the advancement of physics but have no national affiliation.

Applications need to be supported by two Individual Members.

Membership fee for 1983: Sw. Frs, 120 -

4c) Physicists who are members of one of the Member Organizations listed on pages 5 and 6 .

Applications need no supporting signatures.

Membership fee for 1983: Sw.Frs. 40.-

4d) Physicists abroad and physicists visiting Europe who are members of a Collaborating Society (at present, the American Physical Society only). Applications need no supporting signatures.

Membership fee for 1983: Sw.Frs. 50. - = US \$ 25. -

The financial year of EPS corresponds to the calendar year.

An application to join the European Physical Society may be made by completing the following two pages and sending them to the EPS secretariat in Geneva.

The postal address is: European Physical Society

P. O. Box 69

CH-1213 Petit-Lancy 2

Switzerland

\section{Advertising in Europhysics News}

In the Table below are given the rates for advertisements placed in Europhysics News. Normally, advertisements are accepted in the Meetings Issues only when they form part of a series or when there is a dead-line quoted which prevents their being postponed.

Exceptions are also made for conference advertisements giving details supplementary to those quoted in the listings.

For situations vacant advertising a rebate of $25 \%$ is deducted from the normal fee.

EPS Members placing advertisements in Europhysics News receive special rates.

Insertion Rate (Sw.Frs.)

Sw.Frs. 42. - per double column line

Publication: 11 times per year

Printing: Photocomposition in Univers type; offset reproduction.

Advertisements may be composed in standard type without extra charge.

Closing date for order: Third week of month prior to publication. 


\section{EUROPEAN PHYSICAL SOCIETY}

\section{APPLICATION FOR INDIVIDUAL ORDINARY MEMBERSHIP}

1. (Please write in capital letters)

Name:

Date of birth:

Full address:

Highest academic degree:
First name:

Nationality:

2. Professional status (position, institution, specialization)

3. Are you a member of a National Physical Society, Academy or Group which is affiliated to the European Physical Society or of a Collaborating Society? (Please check attached list)
YES
NO

4. If your answer under 3) is YES, of which National Society, Academy, Group or Collaborating Society are you a member?

5. If your answer under 3) is NO, please ask two Individual Ordinary Members of the European Physical Society to sign this form. Kindly print the names and addresses of the referees supporting your application.

Signature of referee:

Signature of referee:

Name and address:

Name and address:

6. Address to which all documentation should be sent (if different from address under 1):

Date and place:

Signature:

Important: Please also fill in over-leaf! 
Full address :

wishes to join the following Divisions and Sections:

DIVISIONS :

ASTRONOMY AND ASTROPHYSICS ATOMIC AND MOLECULAR PHYSICS

COMPUTATIONAL PHYSICS

(Interdivisional Group)

* CONDENSED MATTER

HIGH ENERGY AND PARTICLE PHYSICS

$\square$ NUCLEAR PHYSICS

$\square$ OPTICS DIVISION

PLASMA PHYSICS

QUANTUM ELECTRONICS
SECTIONS:
Solar Physics
Atomic Spectroscopy (EGAS)
Chemical Physics
Electronic and Atomic Collisions
Molecular Physics

Low Temperature Physics

Macromolecular Physics

Magnetism

Metals

Semiconductors and Insulators

Surfaces and Interfaces

* For membership in the Division please note that enrolment into one (or more) Section(s) is necessary according to the rules of the Condensed Matter Division. 
3rd European Symposium on Semiconductor Detectors: New Developments in Si Detectors

R. Klanner, Max-Planck-Inst. f. Physik u. Astrophysik, Föhringer Ring 6, D-8000 München 40

A: 15.9 .83 / Ab: 15.9.83 / PP: 15.9.83/75/inv. English / DM 150. - incl. proc.

\section{4-18 Nov.}

Bratislava, CSSR

Hadron Structure ' 83

- Lukac, Inst. of Physics, Electrophys. Res. Centre,

Slovak Acad. of Sci. 100

Dubravska cesta, CS-84228 Bratislava

\section{0-22 Nov.}

Houston, TX, USA

Meeting of Fluid Dynamics Division (APS)

The American Physical Society,

335 E. 45th Str., New York, NY 10017

20-23 Nov.

San Francisco, CA, USA

General Meeting: American Physical Society

The American Physical Society,

335 E. 45th Str., New York, NY 10017

21-25 Nov.

Granada, Spain

$5^{\circ}$ Asemblea Nacional de Geodesia y Geofisica

Secr. Gen., Instituto Geografico Nacional,

General Ibanez Ibero 3, Madrid-3

A: 21.11.83 / Ab: 15.5.83 / PP: 21.11.83 / Spanish ptas. 1000 . -

22-24 Nov.

Grenoble, France

Dry Plasma Etching in Microelectronics

Mrs. J. Fauvet, 19 rue du Renard, F-75004 Paris

Ab: 20.4.83/PP: 15.9.83/250/Engl., French / FF $1050 .-$

December Trieste, Italy th Trieste Topical Meeting on Elementary Particle Physics

Int. Centre f. Theor. Phys., POB 586, 1-34100 Trieste

5-9 Dec.

Johnsbach, GDR

Int. Seminar on Localization in Disordered Systems

W. Weller, Sektion Physik, Karl-Marx-Universität, Karl-Marx-Platz, DDR-7010 Leipzig

PP / 50 / inv. / English / M 360 .

5-9 Dec.

Philadelphia, PA, USA

10th Symposium on Fusion Engineering

R. G. Mills, Plasma Physics Lab., Princeton Univ. POB 451, Princeton, NJ 08544

7-9 Dec.

Bad Honnef, FRG

Forschungsmanagement in der Physik VIII

W. Heidrich, TTB der KFA Jülich

Postfach 1913, D-5170 Jülich

A: 31.10 .83 / PP / 80 / German / DM 120 - all incl.

8-9 Dec

Cambridge, UK

Molecular and Microstructural Basis of Viscoelasticity and Related Phenomena

Mrs. Y. Fish, The Royal Society of Chemistry, Burlington House, London W1V 0BN

A: Nov. $83 /$ Ab: $29.10 .82 /$ PP: $15.8 .83 / 150 / f 24$.

12-16 Dec.

Florence, Italy

European Regional Astronomy Meeting (IAU)

F. Pacini, Osservatorio Astrofisico di Arcetri,

Largo Enrico Fermi 5, 1-50125 Firenze

Ab: 1.9.83 / Engl. / 300 / Lit. 75000

12-17 Dec.

Miami Beach, FL, USA

8 th Int. Conf. on Infrared and Millimeter Waves

K.J. Button, Nat. Magnet Lab., MIT.

Cambridge, MA 02139

$A b: 1.7 .83 /$ URSI

14-16 Dec.

Oxford, UK

20th Annual Solid State Conference

M.R. Wells, Clarendon Laboratory, Parks Road

Oxford OX13PU
1984

Innsbruck, Austria

Frühjahrstagung Kernphysik (Austrian, German and Swiss Phys. Societies)

W. Breunlich, Inst. f. Radiumforschung u. Kernphysik, Univ. Wien, Boltzmanngasse 3, A-1090 Wien

4-7 Jan.

Tucson, AZ, USA

Protostars and Planets

ab: Nov. 83 / NP / 600 / German, Engl.

27-29 March

Darmstadt, FRG

D. Black, NASA Ames Research Center, Theoret. and Planetary Studies Branch, Code 245-3, Moffett Field, CA 94035

9-12 Jan.

Mérida, Venezuela

Astrometric Techniques (IAU)

H. Eichhorn, SSRB 211, Dept. of Astronomy, Univ, of Florida, Gainesville, FL 32611, USA

16-20 Jan. Toulouse, France

Microwave Signatures in Remote Sensing

Centre d'Etudes Spatiales, Dept, des Affaires Universitaires, 18 av. Edouard-Belin, F-31055 Toulouse Ab: 1.7 .83 / NP / Engl., French / FF 1000.-

23-25 Jan.

New Orleans, LA, USA

Topical Meeting: Optical Fibre Communication

Optical Society of America, 1816 Jefferson Place. N.W. Washington, DC 20036

$\mathrm{Ab}: 2.9 .83 / \mathrm{P}: 2.9 .83$

30 Jan. - 2 Feb.

San Antonio, TX, USA

General Meeting: American Physical Society

The American Physical Society, 335 E. 45th Str. New York, NY 10017

1-13 March

Flavour Mixing in Weak Interactions

Ling-Lie Chan, Physics Dept. 510A,

Brookhaven Nat. Lab., Upton, NY 11973

A: 30.11 .83 / PP / inv. / US \$ 600 . - incl. board, lodging

5-7 March

Topical Meeting: Laser Techniques in Extreme Ultra-

Optical Society of America, 1816 Jefferson Place, N.W. Washington, DC 20036

Ab: 30.11 .83

5-8 March

4th Topical Workshop: Proton-Antiproton Collider Physics

B. Hahn, High Energy Physics Lab., Univ. Bern, Sidlerstr. 5, CH-3012 Bern

12-16 March

48. Physikertagung: Festkörperphysik (DPG

Deutsche Physikalische Gesellschaft, Hauptstrasse 5, D-5340 Bad Honnef

19-23 March

Giessen, FRG

Atomphysik, Massenspektrometrie, Quantenoptik

Deutsche Physikalische Gesellschaft, Hauptstrasse 5, D-5340 Bad Honnef

19-23 March Kiel, FRG

Kurzzeit-, Plasmaphysik, Extraterrestrische Physik

Deutsche Physikalische Gesellschaft,

Hauptstrasse 5, D-5340 Bad Honnef

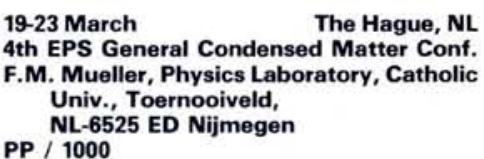

19-23 March The Hague, NL

4th EPS General Condensed Matter Conf.

F.M. Mueller, Physics Laboratory, Catholic

Univ., Toernooiveld,

NL-6525 ED Nijmegen

PP / 1000

21-23 March

Zurich, Switzerland

2nd Int. Symposium on Performance of Computer Com munications Systems

IBM Zurich Res. Lab.

Säumerstr. 4, CH-8803 Rüschlikon

Ab: 1.7.83 / PP: 15.12.83 / Engl., French

21-28 March

Rome, Italy

4th Int. Symposium: Heating in Toroidal Plasmas

H. Knoepfel, ENEA Centre, C.P. 65, I-00044 Frascati

22-24 March

Detroit, MI, USA

Conf. on Defects in Amorphous Materials

W.W. Havens jr., 335 E. 45th Str., New York, NY 10017

26-30 March

Detroit, MI, USA

General Meeting: American Physical Society

The American Physical Society, 335 E. 45th Str. New York, NY 10017
Akustik (DAGA '84)

Deutsche Physikalische Gesellschaft Hauptstrasse 5, D-5340 Bad Honnef

1-4 April

York, UK

Vacuum ' 84 - Technological Aspects of Surface Treatment and Analysis

J.S. Colligon, Thin Film and Surface Res. Centre, Dept. of Electronic and Electr. Engng, Univ. of Salford, Salford M5 4WT

1-5 April York, UK

6th European Conf. on Solid Surfaces (ECOSS-6)

The Meetings Officer, Institute of Physics, 47 Belgrave Square, London SW $1 \times 80 X$

3-5 April

Reno, NV, USA

29th National SAMPE Symposium, Exhibition

M. Smith, National SAMPE Business Office,

POB 613, Azusa, CA 91702

$A b: 1.8 .83 / P: 1.1 .84$

3-6 April

4th Int. Conf. on Energy Options

London, UK

IEE Conf. Dept., Savoy Place, London WC2R OBL

3-6 April

Hetzdorf nr. Dresden, GDR

5 th Int. Seminar on Magnetism (with Dresden Seminar on Theor. Physicsi

K. Elk, Dept. of Physics, Coll. of Transport and Communications, POB 103, DDR-8072 Dresden

A: 15.12 .83 / PP: 3.4 .84 / 50 / inv. / Engl., Russian / M 150. - plus 70. - for accomm.

\section{SUNY}

\section{Stony Brook}

Post-doctoral position is available for an experimental physicist with a background in surface physics.

Experience in any or all of the following fields is desirable: ultra-high vacuum, LEED, AES, UPS, ISS.

Research in surface crystallography will involve correlated experiments at the storage ring (NSLS) and in the laboratory.

Please send résumé, list of publications and references to:

Dr. F. Jona,

Department of Materials

Science and Engineering,

SUNY Stony Brook,

Stony Brook,

N.Y. 11794, U.S.A. 
Working Group on Photographic Problems (IAU)

E. Sim, Royal Observatory, Blackford Hill, Edinburgh EH9 $3 \mathrm{H} \mathrm{J}$

5-6 April Berne, Switzerland

Spring Meeting of Swiss Physical Society

B. Vaucher, Dept. of Physics, Univ. of Fribourg.

$\mathrm{CH}-1700$ Fribourg

Ab: Jan. 84 / PP / 150

\section{9-11 April}

Interfacial Kinetics in Solution

Mrs. Y.A. Fish, The Royal Society of Chemistry, Burlington House, London W1V OBN

A: March 84 / Ab: 15.4.83 / PP: 15.12.83 / 200

9-12 April Garching, FRG

Very Large Telescopes, their Instrumentation and Programs

M.-H. Ulrich, ESO, Karl-Schwarzschild-Str. 2 ,

D-8046 Garching b. München

$9-12$ April

Oxford, UK

3rd Int. Conf. on Mechanical Properties of Materials at High Rates of Strain

J. Harding, Dept. of Engineering Science, Univ. of Oxford, Parks Road, Oxford OX1 3PJ

$\mathrm{Ab}: 11.6 .83 / \mathrm{PP}$

\section{9-13 April}

Knokke, Belgium

SEAS Spring Meeting: Processing Statistical Information

C.J.M. Aarts, SEAS Headquarters, Toernooiveld

NL-6525 Nijmegen

A: 15.3 .84 / Ab: 7.12 .83 / PP: $1.3 .84 / 200$

\section{9-13 April}

Hamburg, FRG

Int. Magnetics Conference (INTERMAG)

W.E. Proebster, IBM Germany,

POB 800880 , D-7000 Stuttgart 80

9-13 April

Eilat, Israel

int. Symposium on Properties and Applications of Metal Hydrides IV

D. Shaltiel, Racah Inst. of Physics, Hebrew University, 91904 Jerusalem

Ab: Sept. 83 / PP: Jan. 84 / US \$150. - incl. proc.

11-13 April

Bradford, UK

Conference on Nuclear Structure Physics

The Meetings Officer, Institute of Physics,

47 Belgrave Square, London SW $1 \times 80 X$

16-20 April

4th Nat. Conf. on Physics (Fisica 84

J.C. Soares, CFNUL, Av. Gama Pinto 2

P-1699 Lisboa, Codex

500 / Port., Spanish, French, Engl.

18-24 April

Monterey, CA, USA

Topical Meeting on Optical Data Storage

Optical Society of America, 1816 Jefferson Place N.W., Washington, DC 20036

Ab: 6.1 .84

\section{6-28 April}

Washington, DC, USA

General Meeting: American Physical Society

The American Phyșical Society, 335 E. 45th Str. New York, NY 10017

\section{7-11 May}

Darmstadt-Seeheim, FRG

Linear Accelerator Conference (LINAC-84)

N. Angert, Ges. f. Schwerionenforschung (GSI),

Postfach 110541, D-6100 Darmstadt

A: 1.12 .83 / Ab: 31.1.84 / PP: 7.5.84 / 150 / inv. DM 200. - incl. proc.

\section{4-18 May}

Debrecen, Hungary

Int. Symposium on Ion-Beam Nuclear Spectroscop

T. Fényes, Inst. of Nuclear Research, Hungarian Acad. of Sciences, Bem tér 18/c, Pf. 51, H-4001 Debrecen

$\mathrm{Ab}: 1.2 .84$

14-18 May

Nagoya, Japan

6th Int. Conf. on Plasma Surface Interactions in Con trolled Fusion

A. Miyahara, Inst. of Plasma Physics, Nagoya Univ. Nagoya 464

21-24 May

St. Louis, MO, USA

Int. Conf, on Plasma Science

T.J. Meene, McDonnell-Douglas Corp.

POB 516, St. Louis, MO 63166

28-30 May

Storrs, CT, USA

Meeting of Electron and Atomic Physics Div. (APS

American Physical Society,

335 E. 45th Str. New York, NY 10017
June

Strasbourg, France

Cool Stars with Excess of Heavy Elements

M. Jaschek, Observatoire de Strasbourg,

11 rue de I'Université, F-67000 Strasbourg

4-6 June

Madison, WI, USA

The Local Interstellar Medium

Y. Kondo, Code 683, NASA-Goddard Space Flight Center, Greenbelt, MD 2077

4-7 June Paris, France

Capteurs 84 - Technologie et Applications

P. Thureau, CIAME, 9 rue Huysmans, F-75006 Paris A: 1.10 .83 / PP: 1.2 .84 / $300 /$ Engl. French

4-7 June

Bruges, Belgium

Int. Symposium: Polymer Alloys - Structure and Properties

R. Fayt, Université de Liège, Institut de Chimie, B-4000 Liège (Sart Tilman)

A: April 34 / Ab: 15.11.83 / PP: 15.2.84 / 150

5-7 June Greenbelt, MD, USA The Origin of Nonradiative Heating / Momentum in Hot Stars

A.B. Underhill, Code 680 , Goddard Space Flight Center Greenbelt, MD 2077

11-15 June Aussois, France

7th Int. Conference on Spectral Line Shapes

F. Rostas, DAPHE, Observatoire de Paris, F-92195 Meudon

A: $1.3 .84 /$ PP: $1.3 .84 / 150$

11-16 June

Lund, Sweden

15th Int. Symposium on Multiparticle Dynamics

B. Andersson, Dept. of Theor. Physics,

Soelvegatan 14A, S-22362 Lund

$100 /$ inv.

17-21 June

Helsinki, Finland

4th Int. Symposium on the Physics of Quark-Gluon Plasmas

T. Toimela, Res. Inst. for Theor. Physics, Siltavuorenpenger 20C, SF-00170 Helsinki 17 $100 /$ inv.

\section{8-20 June}

Princeton, NJ, USA

44th Annual Conf. on Physical Electronics

American Physical Society,

335 E. 45th Str., New York, NY 10017

18-22 June Anaheim, CA, USA

4th Conf. on Lasers and Electro-Optics (CLEO 84)

13th Int. Quantum Electronics Conf. (IOEC 84)

Optical Society of America, 1816 Jefferson Place, N.W. Washington, DC 20036

20-22 June

Dublin, Ireland

3rd Int. Conf. on Boundary and Interior Layers

BAIL III Conf., 39 Trinity College, Univ, of Dublin, Dublin 2

25 June - 7 July Graz, Austria
25th COSPAR Plenary Meeting (and associated events)

25 June - 7 July Graz, Austria
25th COSPAR Plenary Meeting (and associated events)

Z. Niemirowicz, COSPAR, 51 bd de Montmorency, F-75016 Paris

Ab: 1.2.84 / $1000 /$ Engl., French / US \$ 100.-

27-29 June

11th Annual Conf. on Plasma Physics (loP)

H.C. Barr, School of Mathematics and Computer Science, Univ. of North Wales, Bangor, Gwynedd LL57 2UW

\section{June - 3 July}

Int. Conf. on Plasma Physics

M.Q. Tran, Centre de Recherches en Physique des Plasmas, Ecole Polytechnique Fédérale,

21 av. des Bains, CH-1007 Lausanne

PP / 400 / SFr. 300. - incl. proc.

End of June

Essen, FRG

IUTAM Symposium: Influence of Polymer Additives on Velocity and Temperature Fields

K. Kirschke, Deutsche Rheologische Ges. e.V. Unter den Eichen 87, D-1000 Berlin 45

2-6 July

Berlin-West

8 th Int. Congress on Catalysis

A. Benninghoven, Physikal. Inst. der Universität, Dornagkstr. 75, D-4400 Münster
8-14 July

Durham, UK

7th European Symposium on Antiproton Physics

M.R. Pennington, Science Laboratories, Dept. of Physics, University of Durham, South Road, Durham DH1 3LE

Berlin-West

3rd Int. Symposium on Small Particles and Inorganic Clusters

J. Koutecky, Freie Univ. Berlin, Inst. f. Physikal. Chemie, Takusstr. 3, D-1000 Berlin 33

A: 1.5 .84 / Ab: $1.2 .84 / \mathrm{DM} 300$.

15-19 July

Linz, Austria

Laser Processing and Diagnostics: Applications in Electronic Materials

D. Bäuerle, Angewandte Physik, Johannes-Kepler Univ. Linz, A-4040 Linz

Ab: 15.3.84 / PP / 150 / English / öS. 2500.-

15-20 July

5th World Hydrogen Energy Conference

Nat. Res. Council of Canada, Ottawa, Ont. K1A ORG

19-25 July

Leipzig, GDR

22nd Int. Conf. on High Energy Physics

K. Lanius, Inst. f. Hochenergiephysik, Akad. d. Wissenschaften, Platanenallee 6, DDR-1615 Zeuthen 1050 / inv. / IUPAP

23-27 July

Chester, UK

4 th Int. Conf. on Clustering Aspects of Nuclear Structure and Nuclear Reactions

Mrs. S.A. Lowndes, SERC, Daresbury Lab., Daresbury, Warrington WA4 4AD

23-27 July

Zürich, Switzerland

3rd Int. Conf. on Infrared Physics (CIRP 3 )

F. Kneubühl, CIRP - 3, Solid State Physics Lab., ETH, Hönggerberg, $\mathrm{CH}-8093$ Zurich

29 July - 4 Aug.

Bristol, UK

8 th Int. Biophysics Congress

H.C. Watson, Dept, of Biochemistry,

University of Bristol, Medical School,

University Walk, Bristol BS8 1TD

30 July - 3 Aug.

Heidelberg, FRG

10th Int. Conf. on Particles and Nucle

G. zu Putlitz, Physikalisches Institut

der Universität Heidelberg. 900

Philosophenweg 12, D-6900 Heidelberg 1

31 July - 3 Aug.

Espoo, Finland

10th Int. Conf, on Cryogenic Engineering (ICEC 10)

ICEC 10 Secretariat, Low Temperature Laboratory, Helsinki University of Technology, SF-02150 Espoo 15

August Auckland, New Zealand

7th IUPAC Conf, on Physical Organic Chemistry

B.R. Davis, Chemistry Dept, Univ. of Auckland, Private Bag, Auckland

9-18 Aug.

Hamburg, FRG

13th Gen. Ass., Int. Congr. of Crystallography (IUG) H. Saalfeld, Mineral.-Petrograph. Inst., Univ. Hamburg, Grindelallee 48, D-2000 Hamburg 13

13-17 Aug. Stockholm, Sweden

S. Berg, ICTF-6, Inst, of Technology, Uppsala Univ., Box 534, S-75121 Uppsala

13-18 Aug.

Budapest, Hungary

8th European Congress on Electron Microscopy (EUREM 84)

Mr. A. Csanady, Congress Bureau, MOTESZ, POB 32, H-1361 Budapest

13-18 Aug.

Madison, WI, USA

Int. Conf, on Luminescence (ICL 84)

W.M. Yen, Dept, of Physics,

1150 University Ave., Madison, WI 53706

15-22 Aug.

Karlsruhe, FRG

17th Int. Conf. on Low Temperature Physics

$H$. Hinsch, LT 17, Kernforschungszentrum, Postfach 3640, D-7500 Karlsruhe

1000 / English / IUPAP

19-25 Aug.

Lyngby, Denmark

16th Int. Congress of Theor. and Applied Mechanics N. Olhoff, ICTAM 1984, Bldg. 404, Technical Univ. of Denmark, DK-2800 Lyngby

Ab: $6.2 .84 /$ IUTAM 
21-24 Aug.

Brussels, Belgium

Europhysics Conf. on Software Engineering: Methods and Tools in Computational Physics

P. van Binst, Univ. of Brussels, c.p. 230 , 300

d. du Triomphe, B-1050 Brussels

\begin{tabular}{|l|}
\hline 27-31 Aug. \\
6rague, CSSR \\
6th EPS General Conference: Trends in \\
Physics \\
J. Kaczér, Physical Section, Union of Cze- \\
choslovak Mathematicians and Physi- \\
cists, \\
Na Slovance 2, CS-18200 Prague-Liben \\
PP / 700 / English / SFr. 140. - IOM; 180.- \\
mbrs. nat. soc.; 220.- others; 40.- \\
students
\end{tabular}

\section{7-31 Aug.}

Würzburg, FRG

5 th Int. Conf. on Rapidly Quenched Metals

H. Warlimont, Grüner Weg 37, D-6450 Hanau 1

27 Aug. - 1 Sept.

Tokyo, Japan

9th Int. Conf. on Raman Spectroscopy

M. Tasumi, 9th ICORS, Dept. of Chemistry, Faculty of Science, Univ. of Tokyo, Bunkyo-ku, Tokyo 113 IUPAC, IUPAP

28 Aug. - 7 Sept. Varenna, Italy
Int. Course and Workshop on Plasma Astrophysics

28 Aug. - 7 Sept. Varenna, Italy
Int. Course and Workshop on Plasma Astrophysics Int. Course and Workshop on Plasma Astrophysics
C. Paizis, E. Sindoni, Istituto di Fisica, Via Celoria 16. I-20133 Milano

\section{3-6 Sept.}

St. Pölten, Austria

Electronic Structure and Properties of Rare Earth and Actinide Intermetallics

G. Hilscher, Inst, of Exp. Physics,

Tech. Univ. of Vienna, Karlsplatz 13,

A-1040 Vienna

A: 1.3.84 / Ab: 1.3.84 / PP: 1.5.84 / 200 /

SFr. 200.- IOM; 250. - others (incl. proc.)

3-7 Sept.

Darmstadt-Seeheim, FRG

7th Int. Conf. on Atomic Masses and Fundamental Constants (AMCO-7)

E. Roeckl, Ges. für Schwerionenforschung (GSI), Postfach 1105 41, D-6100 Darmstadt

A: 1.5 .84 / Ab: 1.5 .84 / PP: 3.9.84/150/ inv. / approx. DM 200. - incl. proc.

\section{3-7 Sept.}

Budapest, Hungary

Charge Density Waves in Solids

L. Mihaly, POB $49, \mathrm{H}-1525$ Budapest

A: 1.5.84 / Ab: 1.3 .84 / PP: $7.9 .84 / 100 /$ English / US $\$ 80$. -

4-6 Sept.

Leicester, UK

Radicals in Condensed Phases

Mrs. Y.A. Fish, The Royal Society of Chemistry, Burlington House, London W1V OBN

A: Aug. 84 / Ab: 15.9.83 / PP: 15.5.84 / 200

\section{0-14 Sept. Lille, France \\ European Solid State Device Research Conf. \\ E. Constant, Univ. des Sciences et Techni- que de Lille, UER IEEA, bât. P3, F-59655 Villeneuve d'Ascq Cedex PP / 600}

10-14 Sept.

Oak Ridge, TN, USA

5 th Int. Symposium on Capture Gamma Ray Spectroscopy and Related Topics

S. Raman, Oak Ridge Nat. Lab., Physics Division, POB X, Oak Ridge, TN 37830

10-15 Sept.

Zurich, Switzerland

22nd Ampère Congress

R. Kind, Lab. Solid State Physics,

ETH Hönggerberg, CH-8093 Zurich

12-19 Sept.

London, UK

10th Int. Conf. on Plasma Physics and Nuclear Fusion Research

R. Najar, Conf. Service, IAEA, POB 100, A-1400 Vienna

17-21 Sept.

Manchester, UK

9th European Conf. on Thermophysical Properties

The Meetings Officer, The Institute of Physics, 47 Belgrave Square, London SW $1 \times 80 \times$

\section{3-28 Sept.}

Balatonfüred, Hungary

12th Int. Hot Atom Chemistry Symposium

L. Vasaros, Central Res. Inst. f. Physics,

POB 49, H-1525 Budapest
24-28 Sept.

Garmisch-Partenkirchen, FRG

SEAS Anniversary Meeting

C.J.M. Aarts, SEAS Headquarters,

Toernooiveld, NL-6525 ED Nijmegen

A: 1.9 .84 / Ab: 15.5 .84 / PP: $1.9 .84 / 300$

25-28 Sept.

Dresden, GDR

Int. Conf. on Energy Pulse Modification of Semicon ductors and Related Materials

H.U. Jäger, Akad. d. Wissenschaften der DDR

Zentralinstitut $f$. Kernforschung Rossendorf,

POB 19, DDR-8051 Dresden

A: 31.3 .84 / Ab: 1.2 .84 / PP: 31.10 .84 / 200 / English / M 350 . -

$1-30 \mathrm{ct}$

Utrecht, NL

Triennial European General Meeting on Solar Physics: Hydromagnetism of the Sun

P. Hoyng, Sterrewacht Sonnenborgh, Zonnenburg 2. PP / 150

9-12 Oct.

ECOOSA ' 84

Amsterdam, NL

J. Langelaar, Foundation Quantoptica

Lab. Phys. Chem., Nieuwe Achtergracht 127 NL-1018 ES Amsterdam

P: 1.5.84/350/Advance fee hFI. 250.-: current $325 .-$; students 50 . -

18-20 Oct.

Nashville, TN, USA

Meeting of Nuclear Physics Div. (APS)

American Physical Society,

335 E. 45th Str., New York, NY 10017

24-29 Oct.

Takarazuka, Osaka, Japan

9 th Int. Conf. on Infrared and Millimetre Waves

H. Yoshinaga, Dept. of Applied Physics, Osaka Univ. Yamada-Oka, Suita, Osaka 565

27 Oct. - 3 Nov.

Meeting of Plasma Physics Div. (APS)

American Physical Society,

335 E. 45th Str., New York, NY 10017

29 Oct. - 2 Nov.

San Diego, CA, USA

Annual Meeting of Optical Society of America

Optical Society of America, 1816 Jefferson Place,

N.W., Washington, DC 20036

18-20 Nov.

Providence, RI, USA

Meeting of Fluid Dynamics Div. (APS)

American Physical Society,

335 E. 45th Str., New York, NY 10017

12-13 Dec.

Cambridge, UK

Molecular Electronic Structure Calculations: Methods and Applications

Mrs. Y.A. Fish, The Royal Society of Chemistry, Burlington House, London W1V OBN

A: Nov. 84 / Ab: 31.8.84 / PP: 15.8.84 / 150

16-21 Dec.

Int. Conf. on Engineering Ceramics

Jerusalem, Israe

Ms. Sh. Cahana, Nat. Council for Research and DevePP

opment, Prince Minister's Office, Jerusalem 91000

\section{5}

Int. Conference on Phonon Physics

Budapest, Hungary

N. Kroo, POB 49, H-1525 Budapest

400

15-19 April Amsterdam, NL

2nd European Conf. on Atomic and Molecular Physics

M.J. van der Wiel, FOM Inst. f. Atomic and Molecular Physics.

Kruislaan 407, NL-1098 S.J Amsterdam

Ab: Jan. 85 / hFI, 200,

September

9th European Crystallographic Meeting

Turin, Italy

M. Catti, Istituto di Mineralogia e Cristallografia dell' Università, Via Massimo 22, 1-10123 Torino

\section{Schools 1983}

Scholarships for sponsored schools at Erice are available on application to the EPS Secretariat.

19-28 Aug. San Miniato, Italy - Int. School for Intermediate Energy Nuclear Physics

22 Aug. - 2 Sept. Aspenäsgarden, Sweden Europhysics Summer School: Chemisorption and Surface Reactions

B. Lundqvist, Chalmers University, S-41296 Göteborg

PP / 70 / sKr. 3365. - IOM; 3430. - mbrs 4b 3500.- others (incl. board \& lodging)

25 Aug. - 6 Sept.

Poiana Brasov, Romania

- Brasov Int. Summer School: Theoretical Aspects of Critical Phenomena

2-10 Sept. Pisa, Italy
50 Ecole d'Eté Méditerranéenne sur les Etats de Sur-

2-10 Sept. Pisa, Italy
50 Ecole d'Eté Méditerranéenne sur les Etats de Surface et la Liaison Chimique: Physisorption, Chemisorption and Adhesion

3-10 Sept.

Davos, Switzerland

-5th Int. Summer School on Crystal Growth and Mate rials Research

4-16 Sept.

Cambridge, UK

Advanced Study Inst.: Physics and Chemistry of Electrons and Related lons in Condensed Matter

J.V. Acrivos, Dept. of Chemistry, San José State Univ. San José, CA 95192 USA

4-16 Sept.

Erice, Italy

Int. School of Quantum Electronics: Laser Applications to Biology and Medicine

S. Martellucci, c/o Mrs. M. Fiorini, ENEA, c.p. 65 1-00044 Frascati

A: $\mathbf{3 0 . 6 . 8 3} / 90$ / US $\$ 650 .-$ all incl.

430 Sept

USSR

Inter-regional Training Course, Study Tour: Neutron Physics, Nuclear Data Measurements with Accelerators, Research Reactors

IAEA, POB 100, A-1400 Vienna

English, Russian

5-9 Sept.

Marseille, France

15e Ecole d'Eté de Physique des Particules

E. Perret, IN2P3, 20 rue Berbier du Mets. F-75013 Paris

5-16 Sept.

- Quarks, Leptons and Beyond

Munich, FRG

5-17 Sept.

Mikolajki, Poland

15th Int. Summer School in Nuclear Physics

K. Siwek-Wilczynska, Inst. of Experim. Physics,

Warsaw Univ., 69 Hoza St., PL-00-681 Warsaw

6-16 Sept.

Maria Laach, FRG

Elektroschwache Wechselwirkung: Grundlagen, neue Ergebnisse, Vorbereitungen für LEP Experimente J.K. Bienlein, DESY, Notkestr. 85, D-2000 Hamburg 52 50 / inv. / German / DM 300.-

7-17 Sept.

Varenna, Italy

- Course and Workshop: Mirror-Based and Field-Reversed Approaches to Magnetic Fusion

12-16 Sept.

Bombannes, France

Ecole Joliot-Curie de Physique Nucléaire: Structure nucléaire aux frontières de la stabilite

E. Perret, IN2P3, 20 rue Berbier du Mets,

$$
\text { F-75013 Paris }
$$

French

12-16 Sept.

St. Andrews, UK

2nd British Postgraduate School in Nuclear Physics

12-22 Sept.

4th Int. School of Coherent Optics

12-30 Sept.

Workshop IV on Nonlinear Stability

18-24 Sept.

Hajduszoboszlo, Hungary

2nd Int. Summer School on Processes of Thin Film Formation

18 Sept. - 2 Oct. Kupar - Dubrovnik, YU

- Int. School of Elementary Particle Physics 


\section{Sept. -8 Oct.}

Autumn College on Soil Physics

Trieste, Italy

Int. Centre f. Theor. Phys., POB 586, 1-34100 Trieste

24-30 Sept.

2nd Petra School of Physics

A. Salem Saleh, Dept. of Physics,

Yarmouk Univ., Irbid, Jordan

3-14 Oct.

DPG - Schule für Physik

Bad Honnef, FRG

Anwendungen (3-7.10

Teil II: Aufbau u. Entwicklung der Sterne (10-14.10.)

J. Debrus, Physikzentrum Bad Honnef, Hauptstrasse 5 D-5340 Bad Honnef

A: 1.7.83 / NP / 60/German / DM 250. - (I) 220. - (II) all incl.

\section{1-21 Oct.}

CERN, Geneva, $\mathrm{CH}$

CERN Accelerator School Course: Antiprotons fo Colliding Beam Facilities

Mrs. F. Têtu, DG Office, CERN Accelerator School $\mathrm{CH}-1211$ Geneva 23

A: 1.9 .83 / PP / 120 / SFr. 100. - incl. proc.

17-21 Oct.

Lisbon, Portuga

5th Autumn School of Physics: Field Theory and Ele mentary Particles

J.C. Romao, IFM,

Av. Gama Pinto 2, P-1699 Lisbon Codex

NP / 40 / English

17 Oct. - 11 Nov.

Trieste, Italy

Nuclear Physics Workshop

Int. Centre f. Theor. Phys., POB 586, 1-34100 Trieste

Int. School of Radiation Damage and Protection (A

Rindi): Biological Effects and Dosimetry of Nonionizing Radiation - Static and ELF Electromagnetic Fields

M. Grandolfo, Ist. Superiore Sanità

Viale Regina Elena 299, I-00161 Roma

\section{4}

16 Jan. - 3 Feb.

Workshop Trieste, Italy Int. Centre f. Theor. Phys., POB 586, 1-34100 Trieste

7 Feb. - 30 March

Trieste, Italy

Winter College on Fundamental Nuclear Physics

Int. Centre f. Theor. Phys., POB 586, 1-34100 Trieste A: 31.8 .83

20 Feb. - 1 March

Schladming, Austria

3. Int. Universitătswochen f. Kernphysik

Org. Comm., Inst. f. Theor. Physik, Universitätplatz 5 , A-8010 Graz

A: $6.2 .84 / \mathrm{PP} / 150 /$ English

7-19 April

Int. School of Crystallography: Direct Methods for Solving Crystal Structures

T.L. Blundell, Dept. of Crystallography,

Birkbeck College, Univ. of London,

Malet Str, London WC1E 7HX, UK

A: 31.10 .83 / 65 / SFr. 1250. - approx. all inci.

8-20 April

Erice, Italy

Int. School of Nuclear Physics: Nuclear and Subnuclear Degrees of Freedom and Lepton Nucleus Scattering

A. Faessler, Universität Tübingen, Inst. f. Theor. Physik, Auf der Morgenstelle 14,

D-7400 Tübingen

A: $1.3 .84 / 120 /$ US $\$ 600 .-$ all incl.

$\begin{array}{lr}26 \text { April - } 22 \text { June } & \text { Trieste, Italy } \\ \text { Spring College on Crystalline Semiconducting Materials }\end{array}$

$\begin{array}{lr}26 \text { April - 22 June } & \text { Trieste, Italy } \\ \text { Spring College on Crystalline Semiconducting Materials }\end{array}$ and Devices

Int. Centre f. Theor. Phys., POB 586, I-34100 Trieste

11-24 June

1984 CERN School of Physics

D.A. Caton, Scientific Conference Secretariat, CERN, CH-1211 Geneva 23, Switzerland

A: $23.3 .84 / 80$

1-15 July

Erice, Italy

Int. School of Material Science and Technology: Surface Dynamical Phenomena

B. Feuerbacher, Inst. f. Raumsimulation,

DF Linder Höhe, Postfach 906058, D-5000 Köln 90

September

1984 CERN School of Computing

Mrs. I. Barnett, Scientific Conference Secretariat CERN, $\mathrm{CH}-1211$ Geneva 23, Switzerland

21-30 Sept.

Varna, Bulgaria

3rd Int. School on Condensed Matter Physics: Nonlinear Phenomena in Solids - Modern Topics

A.F. Vavrek, Inst. of Solid State Physics, Lenin bul. 72 , BG-1184 Sofia

A: 31.5.84 / Ab: 31.5 .84 / PP / 100 / Engl., Russian / US $\$ 60 .-$

\section{EPS Sponsorship of Schools and Conferences}

Application for sponsorship of Schools or Conferences should be addressed to the EPS Secretariat in Geneva normally at least nine months before the event. Conditions governing acceptance are that the event must be truly international from the point of view of both attendance and the programme committee, and it must be an event that fulfils a clear need and is of high quality in regard to both speakers and contributions. There must also be no restrictions on attendance other than those dictated by scientific considerations.

EPS Divisions, Sections and Group Astronomy and Astrophysics Division Solar Physics Section

Atomic and Molecular Physics Division

Atomic Spectroscopy Section

Chemical Physics

Electronic and Atomic Collisions

Molecular Physics

Computational Physics Group

Comdensed Matter Division

Low Temperature Physics Section

Macromolecular Physics

Magnetism

Metal Physics

Semiconductors and Insulators

Surfaces and Interfaces

High Energy \& Particle Physics Division

Nuclear Physics Division

Optics Division

Optics Division
Plasma Physics Division

Plasma Physics Division
Europhysics News is the official journal of the European Physical Society which comprises 29 National Societies, Academies and Groups, over 3000 Individual Members and 63 Associate Members. Governing bodies of EPS are the General Meeting, Council and an dies of EPS are the General Meeting, Council and an elected Executive Committee responsible for detailed
policy. EPS promotes the collaboration of physicists throughout Europe, organising and harmonising conferences, and promotes international exchanges in physics including participation in research and teaching sics including participation in research and teaching
activities abroad and attendance at schools. EPS publishes in addition to EN, Europhysics Conference Abstracts, E. Ed. News and, in collaboration with The Institute of Physics (UK), the European Journal of Physics. Individual Members receive EN free of charge (price to institutions: Sw.Fr. 82.-/a), rebates on the (price to institutions: Sw.Fr. $82 .-/ a$ ), rebates on the
price of many publications and on conference fees. Annual EPS membership fee for Individual Members who belong to one of the EPS member societies for 1983 is: Sw.Fr. 40.-; for independent members: Sw.Fr. 120.

\section{European Journal of Physics}

In the report on the EPS Council meeting published in the April issue of Europhysics News, a brief mention was made of the growing circulation of the European Journal of Physics which had resulted in the journal becoming self supporting. Experience with the first three volumes that have now appeared has brought to light two unexpected aspects.

In the first place, it seems that in Europe there is a certain reluctance on the part of lecturers in the higher educational establishments to share their teaching experience with others, and to put down on paper the techniques and methods they have devised and which have proved successful in practice. In part this is, of course, due to the heavy work load that most are carrying which results in their concentrating either on their teaching as such, or on their research and the publication of the results of that research. In part, however, it would seem that an excess modesty prevails and many do not appreciate that what they are doing would be of real interest to their colleagues.

The message from the Editorial Board is therefore not to be bashful. They are keen to receive contributions covering all aspects of the teaching of physics at undergraduate level - even to learn of schemes which have met unexpected pitfalls. They would also like to receive more contributions of a reflective nature which discuss the philosophical aspects of physics.

This raises the second point which is that the purpose of the journal is not everywhere fully understood. The European Journal of Physics is designed to be the European communication medium of all concerned with the intellectual advancement of physics - the "common room" of those engaged in developing the next generation of physicists.

Contributions should be sent to:

The Managing Editor,

European Journal of Physics

Techno House, Redcliffe Way,

Bristol BS1 6NX, England

Editor: E.N. Shaw

Meetings Compilation: W.S. Newman

Editorial Board

K. Appert, A. Baratoff, G.J. Béné,

G.R. Macleod, A. Maeder, J. Muller

Editorial and Advertising Office at the EPS Secretariat.

Address: EUROPEAN PHYSICAL SOCIETY P.O. Box 69 . CH-1213 Petit-Lancy 2 Switzerland

Telephone: Geneva (22) 931130

Telex: 423455 dema $c h$

Cables: europhys genève

Printed by: Pfirter frères SA

CH-1213 Petit-Lancy/Switzerland 